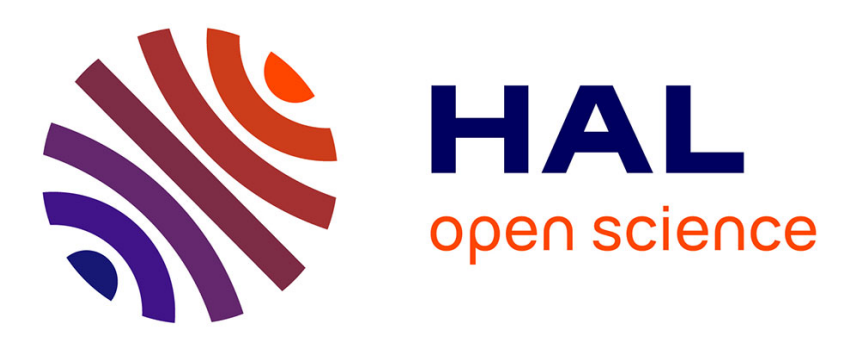

\title{
Les stèles mérovingiennes, premiers témoignages des communautés chrétiennes rurales d'Île-de-France
}

Stéphane Ardouin

\section{To cite this version:}

Stéphane Ardouin. Les stèles mérovingiennes, premiers témoignages des communautés chrétiennes rurales d'Île-de-France. Archéologie médiévale, 2017, 47 (47), pp.53-70. 10.4000/archeomed.2753 . halshs-03517004

\author{
HAL Id: halshs-03517004 \\ https://shs.hal.science/halshs-03517004
}

Submitted on 7 Jan 2022

HAL is a multi-disciplinary open access archive for the deposit and dissemination of scientific research documents, whether they are published or not. The documents may come from teaching and research institutions in France or abroad, or from public or private research centers.
L'archive ouverte pluridisciplinaire HAL, est destinée au dépôt et à la diffusion de documents scientifiques de niveau recherche, publiés ou non, émanant des établissements d'enseignement et de recherche français ou étrangers, des laboratoires publics ou privés.

\section{(ㄷ)(1) $\$$}

Distributed under a Creative Commons Attribution - NonCommerciall 4.0 International 


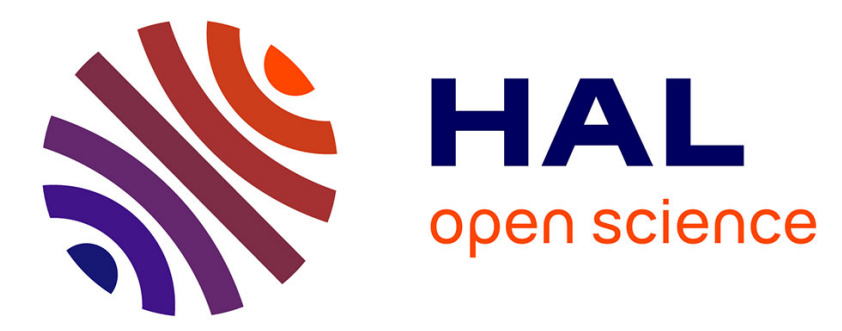

\section{Les stèles mérovingiennes, premiers témoignages des communautés chrétiennes rurales d'île-de-France}

Stéphane Ardouin

\section{To cite this version:}

Stéphane Ardouin. Les stèles mérovingiennes, premiers témoignages des communautés chrétiennes rurales d'Île-de-France. Archéologie Médiévale, CRAHAM, 2017, 47 (47), pp.53-70. 10.4000/archeomed.2753 . halshs-03517004

\section{HAL Id: halshs-03517004 https://halshs.archives-ouvertes.fr/halshs-03517004}

Submitted on 7 Jan 2022

HAL is a multi-disciplinary open access archive for the deposit and dissemination of scientific research documents, whether they are published or not. The documents may come from teaching and research institutions in France or abroad, or from public or private research centers.
L'archive ouverte pluridisciplinaire HAL, est destinée au dépôt et à la diffusion de documents scientifiques de niveau recherche, publiés ou non, émanant des établissements d'enseignement et de recherche français ou étrangers, des laboratoires publics ou privés.

\section{다)(1) $(5$}

Distributed under a Creative Commons Attribution - NonCommerciall 4.0 International 


\title{
Les stèles mérovingiennes, premiers témoignages des communautés chrétiennes rurales d'île-de-France
}

\author{
Stéphane ARDOUIN*
}

Mots-clés : Île-de-France, antiquité tardive, haut Moyen Âge, nécropole, stèle funéraire, épitaphe, remploi

Résumé : De nombreuses stèles funéraires mérovingiennes ont été mises au jour en île-de-France, principalement au nord-ouest de cette région, à la croisée des diocèses de Rouen, Paris, Évreux et Chartres. Bien qu'inscrites dans une tradition d'origine antique, elles s'en distinguent par leur nombre. Presque toutes anépigraphes, elles sont très fréquemment gravées de symboles qui varient d'une nécropole à l'autre. Les nombreuses gravures de croix, dans toute leur diversité, évoquent la représentation de symboles chrétiens, indiscutables dans certains cas, mais également des motifs plus géométriques qui rappellent les décors des sarcophages de plâtre, très fréquents en île-de-France. Datées des Vle et vII siècle, souvent découvertes en remplois, les stèles constituent, avec de très rares épitaphes et quelques vestiges d'édifices, les manifestations les plus anciennes attribuables aux premières communautés chrétiennes rurales de la région.

Keywords: Île-de-France, Late Antiquity, High Middle Ages, grave site, funerary steles, epitaph

Abstract: Merovingian steles as early evidence of rural Christian communities in île-de-France. Numerous Merovingian funerary steles have been excavated in Île-de-France, most notably in the North-Western part of this region, in an area encompassing the dioceses of Rouen, Paris, Evreux and Chartres. Inherited from an antique tradition, the Merovingian exemples are however more numerous. Most of them are bare of any inscription, though they are frequently ingraved with symbols, which vary from one grave site to the other. Numerous cross figures are found, variable in shapes and forms, alluding to Christian symbols, sometimes in a definitive way. Some of the motifs are more geometric, similar to the patterns found on plaster sarcophagi from île-de-France. Dated from the 6th and 7th c. and often found in a different context than their original purpose, these steles are some of the earliest traces linked to the first rural Christian communities of the area, along with a few inscriptions and remaining vestige of buildings.

Schlüsselwörter: Île-de-France, Spätantike, Frühmittelalter, Nekropole, Grabstele, Grabschrift

Zusammenfassung: Die merowingerzeitlichen Stelen als erste Zeugnisse der ländlichen christlichen Gemeinden der île-de-France. In der île-de-France wurden viele merowingerzeitliche Grabstelen gefunden, hauptsächlich im Nordwesten dieser Gegend, im Grenzbereich der Diözesen Rouen, Paris, Evreux und Chartres. Zwar sind sie in einer antiken Tradition verwurzelt, aber sie fallen auf durch ihre große Anzahl. Sie sind fast alle anepigraphisch, aber tragen sehr häufig eingegrabene Symbolen, die sich von einer Nekropole zum anderen unterscheiden. Dabei sind die zahlreichen und sehr verschiedenen eingemeißelten Kreuze in manchen Fällen eindeutig als christliche Symbole zu deuten; aber in anderen Fällen ist eher an rein geometrische Muster zu denken, die an die Ornamentik der in der île-de-France sehr zahlreichen Gipssarkophage erinnern. Diese Stelen, die vom 6. und 7. Jh. datieren und oft wiederverwendet gefunden wurden, sind - mit den sehr seltenen Grabschriften und einigen Resten von Kirchenbauten - die ältesten Spuren, die die ersten christlichen Gemeinden des platten Landes in der île-de-France hinterlassen haben.

* Chercheur associé à l'UMR 5138 Archéologie et Archéométrie, Service archéologique de la ville de Lyon. 
Des stèles funéraires ont régulièrement été mises au jour au sein des nécropoles mérovingiennes du nord-ouest de l'Îlede-France comme dans celles de l'Oise ${ }^{1}$ et de l'Aisne ${ }^{2}$, au sud de la Picardie et de la Champagne. Elles se raréfient autour de ces secteurs géographiques qui semblent avoir privilégié la pierre pour la signalisation des sépultures en surface. L'usage de stèles funéraires en pierre n'est pas une nouveauté mérovingienne. Fréquente dès l'Antiquité, cette pratique funéraire s'est manifestée par la réalisation de grandes stèles associant régulièrement des inscriptions (épitaphes ou simples dédicaces abrégées aux dieux Mânes) à des représentations figurées sculptées évoquant les défunts sur la partie haute de la stèle. Si certaines stèles mérovingiennes pourraient dériver de modèles romains tardifs ${ }^{3}$, aucune continuité de ces usages n'a été observée au sein des mêmes zones funéraires. Les stèles funéraires romaines sont ainsi plutôt rares dans ces mêmes régions et fréquemment limitées aux zones urbaines. En Île-de-France, en effet, des stèles assez nombreuses ont été découvertes dans les nécropoles urbaines ou périurbaines de Paris $^{4}$, Meaux 5 , Melun ${ }^{6}$ et dans l'agglomération secondaire d'Epiais-Rhus ${ }^{7}$. Seuls un exemplaire mis au jour à SaintGermain-lès-Arpajon $^{8}$ (Essonne) et deux découverts à Avon ${ }^{9}$ (Seine-et-Marne) sont recensés en zone rurale.

Limitées dans l'espace, les stèles mérovingiennes le sont également dans le temps, n'ayant été utilisées que sur une courte période, entre la fin $\mathrm{du}^{\mathrm{e}}$ et la fin du VII ${ }^{\mathrm{e}}$ siècle. Anépigraphes, elles comportent fréquemment des décors chrétiens. La stèle est avec l'épitaphe le seul usage funéraire susceptible de distinguer les chrétiens des païens au IV siècle. $\mathrm{Si}$, durant la période mérovingienne, les cimetières mérovingiens sont potentiellement tous chrétiens ${ }^{10}$, ces représentations sur les stèles demeurent bien souvent les premières manifestations visibles des communautés chrétiennes en zone rurale.

\section{PRÉSENTATION DES STÈLES MISES AU JOUR EN ÎLE-DE-FRANCE}

\subsection{STÈLES ET RITUELS DE FUNÉRAILLES}

La stèle funéraire, monolithe dressé verticalement à l'emplacement d'une sépulture, permet sa signalisation en surface. Elle participe, comme l'épitaphe, de la commémoration des défunts et personnalise la sépulture en la distinguant des sépultures voisines au sein d'une nécropole. À ce titre, elle pourrait être susceptible de révéler le rang social du défunt ou

\footnotetext{
1. Flèche Mourgues 1998.

2. Poulain 1984.

3. SALIN 1952, p. 75.

4. BusSON 1998.

5. Delattre, Magnan 1998; Griffisch, Magnan, Mordant 2008.

6. Benhadou (dir.) 1990

7. Deux fragments de stèles mis au jour (WABont, Abert, Vermeersch 2006, p. 246).

8. Naudet 2004, p. 225, fig. 120.

9. Griffisch, Magnan, Mordant 2008, p. 245.

10. Dierkens, PÉRIN 2011.
}

des individus ayant organisé les funérailles. Les motifs figurés pourraient aussi révéler la dévotion de certains défunts.

La diffusion du christianisme au sein des villes mérovingiennes s'est caractérisée par la multiplication des édifices cultuels, dont on a conservé de nombreux témoignages. Plus rares et moins ostentatoires dans les campagnes, ces édifices sont aussi plus difficiles à mettre en évidence. La recherche des manifestations religieuses en zone rurale passe donc par l'exploration des nécropoles. À l'exception de rares incinérations, difficilement représentatives de traditions chrétiennes ${ }^{11}$, peu de pratiques funéraires mérovingiennes pourraient être considérées comme païennes, le paganisme ne survivant plus à la période mérovingienne que sous la forme de pratiques traditionnelles plus folkloriques que religieuses ${ }^{12}$. Il n'existait vraisemblablement pas de rituel de funérailles fixé par l'Église pour les chrétiens de l'Antiquité tardive avant le $\mathrm{VII}^{\mathrm{e}}$ ou le VIII $^{\text {e }}$ siècle ${ }^{13}$. Les pratiques funéraires s'organiseraient donc durant cette période en dehors des structures religieuses proprement dites ${ }^{14}$. Les évocations des croyances religieuses figurant sur les stèles sont donc a priori et sans surprise en relation avec le christianisme, seule religion officielle en Gaule mérovingienne.

Si des stèles ou épitaphes chrétiennes comportent encore des formules anciennes typiquement païennes telles que « $D$ (is) $M$ (anibus) ${ }^{15}$, signe que plusieurs croyances ou traditions pouvaient encore coexister dans un monde devenu institutionnellement chrétien après la conversion de Clovis, aucune n'est recensée en Île-de-France alors que des motifs indiscutablement chrétiens sont fréquents sur les monuments issus des nécropoles rurales.

Les stèles funéraires en pierre ont souvent été négligées dans les fouilles anciennes de cimetières mérovingiens et peu d'entre elles ont été inventoriées, conservées ou publiées ${ }^{16}$. En France, peu d'études sur ces monuments peuvent être mises en avant. L'une des plus importantes est la classification chronotypologique des stèles mises au jour en Picardie, réalisée par M.-P. Flèche Mourgue ${ }^{17}$. L'Île-de-France a bénéficié des travaux de J. Sirat, qui entreprit à partir des années 1960 de recenser les stèles du Vexin français. Les autres secteurs de l'Île-de-France, où les stèles découvertes sont étrangement peu nombreuses, n'ont pas fait l'objet de recensements ni d'études synthétiques.

\subsection{RÉPARTITION DES STÈLES EN ÎLE-DE-FRANCE}

Le recensement des stèles franciliennes intègre essentiellement les stèles publiées, qui ont pu être ponctuellement complétées par le dépouillement de rapports d'intervention ${ }^{18}$.

\footnotetext{
11. Rebillard 2003, p. 96-101.

12. Dierkens, PÉrin 2011.

13. Rebillard 2003, p. 40-49; Treffort 1996, p. 165.

14. Young 1991, p. 115.

15. MacMullen 1998, p. 153.

16. Young 1991, p. 114.

17. Flèche Mourgues 1998.

18. Une première approche de ce recensement, amorcé lors d'un travail universitaire (ARDouin 2008), a été présentée dans le cadre d'un poster lors des Journées internationales d'archéologie mérovingienne tenues au Luxembourg en 2010 (ARDouin à paraître).
} 


\begin{tabular}{|c|c|c|c|c|c|c|c|c|c|}
\hline & Commune & Lieu-dit & Département & Diocèse & $\begin{array}{l}\mathrm{Nb} \text { minim } \\
\text { de stèles }\end{array}$ & $\begin{array}{c}\text { Nb minim de } \\
\text { stèles } \\
\text { décorées }\end{array}$ & $\begin{array}{c}\text { Nb minim de } \\
\text { sépultures }\end{array}$ & $\begin{array}{c}\text { Datation } \\
\text { nécropole }\end{array}$ & Remarques \\
\hline 1 & Paris & Saint-Germain-des-Prés & Paris & Paris & 1 & 1 & & $I^{e}-$ & \\
\hline 2 & Paris & Saint-Marcel & Paris & Paris & 1 & 1 & & IV & \\
\hline 3 & Baulne & Saint-Fiacre & Essonne & Sens & ind & ind & & Indéterminé & Indice \\
\hline 4 & Grigny & & Essonne & Paris & ind & ind & 65 & & \\
\hline 5 & Andresy & Les Barils & Yvelines & Paris & 15 & 9 & 492 & $V^{e}-V I I I^{e}$ & \\
\hline 6 & Epône & Les Culs Chevets & Yvelines & Chartres & 9 & 3 & 300 & $\left\|V^{e}-V\right\|^{e}$ & \\
\hline 7 & Gaillon-sur-Montcient & La garenne & Yvelines & Rouen & 50 & 13 & 220 & $V^{e}-V I I I^{e}$ & \\
\hline 8 & Guitrancourt & La Grippe & Yvelines & Rouen & 6 & 3 & 78 & $V I^{e}-V I I^{e}$ & \\
\hline 9 & Herbeville & Les Closeaux & Yvelines & Chartres & 1 & 1 & 0 & Indéterminé & découverte isolée \\
\hline 10 & Maule & Pousse-Motte & Yvelines & Chartres & 52 & 19 & 807 & $V^{e}-V I I I^{e}$ & \\
\hline 11 & Rosny-sur-Seine & Saint-Lubin & Yvelines & Chartres & 1 & 1 & Ind & $V l^{e}-$ & \\
\hline 12 & Septeuil & La Pierre Bât & Yvelines & Chartres & 6 & 1 & 41 & $V l^{e}-V I I^{e}$ & \\
\hline 13 & Septeuil & Les Grands Bilheux & Yvelines & Chartres & 1 & 1 & 0 & & découverte isolée \\
\hline 14 & Noisy-le-Grand & Les Mastraits & Seine-Saint-Denis & Paris & 23 & 0 & 219 & $V I^{e}-V I I I^{e}$ & \\
\hline 15 & Fresnes & La Tour aux Chartiers & Val-de-Marne & Paris & 1 & 1 & 84 & $\left\|V^{e}-X\right\|^{e}$ & \\
\hline 16 & L'Haÿ-les-Roses & Les Hautes-Bruyères & Val-de-Marne & Paris & 2 & 1 & 60 & $V l^{e}-V I I^{e}$ & \\
\hline 17 & Ableiges & Les Buis & Val d'Oise & Rouen & 22 & 18 & 200 & $\left\|V^{e}-V\right\|^{e}$ & \\
\hline 18 & Amenucourt & Val Perron & Val d'Oise & Rouen & 2 & 2 & 40 & $V l^{e}-V I^{e}$ & \\
\hline 19 & Baillet-en-France & Vieille-Eglise & Val d'Oise & Paris & 1 & 1 & 5 & $V l^{e}-\mathrm{Xl}^{\mathrm{e}}$ & \\
\hline 20 & Banthelu & Le Moulin à Vent & Val d'Oise & Rouen & 39 & 18 & 165 & $V I^{e}-X I I^{e}$ & \\
\hline 21 & Beaumont-sur-Oise & Saint-Léonor & Val d'Oise & Beauvais & 1 & nd & 136 & & \\
\hline 22 & Condécourt & Le Moulin à Vent & Val d'Oise & Rouen & 5 & 2 & 26 & $V l^{e}-V I I^{e}$ & \\
\hline 23 & Genainville & Les Vaux de la Celle & Val d'Oise & Rouen & 1 & 0 & 0 & & Site de production \\
\hline 24 & Genainville & La Couture & Val d'Oise & Rouen & 2 & 0 & 5 & Indéterminé & \\
\hline 25 & Guiry-en-Vexin & La Nourotte & Val d'Oise & Rouen & 2 & 1 & 255 & $V^{e}-V I I^{e}$ & \\
\hline 26 & Guiry-en-Vexin & Sur-les-Quais & Val d'Oise & Rouen & 1 & 0 & 49 & $\mathrm{VI} \mathrm{I}^{\mathrm{e}}-\mathrm{VIII} \mathrm{I}^{\mathrm{e}}$ & Nombreuses réductions \\
\hline 27 & Longuesse & La Grande Pièce & Val d'Oise & Rouen & 5 & 3 & 47 & Indéterminé & Nombreuses réductions \\
\hline 28 & Louvres & Saint-Rieul & Val d'Oise & Paris & 1 & nd & 400 & $v^{e}-$ & \\
\hline 29 & Magny-en-Vexin & Blamecourt & Val d'Oise & Rouen & 3 & 2 & 10 & Indéterminé & \\
\hline 30 & Magny-en-Vexin & Collège $\mathrm{Cl}$. Monet & Val d'Oise & Rouen & 1 & 1 & 100 & Indéterminé & \\
\hline 31 & Mériel & Stors & Val d'Oise & Paris & 7 & 1 & 21 & Indéterminé & \\
\hline 32 & Nucourt & Le Vieil Aître & Val d'Oise & Rouen & 2 & 1 & 50 & $\mathrm{~V}^{\mathrm{e}}-\mathrm{VII}{ }^{\mathrm{e}} ?$ & \\
\hline 33 & Parmain & La Butte de Montareine & Val d'Oise & Beauvais & 1 & 1 & & & \\
\hline 34 & Presles & La Côte-des-Trépassés & Val d'Oise & Beauvais & 3 & 3 & & Indéterminé & \\
\hline 35 & Santeuil & Les Epagnes & Val d'Oise & Rouen & 17 & 12 & 193 & $V^{e}-X^{e}$ & \\
\hline Total & & & & & 285 & 121 & & & \\
\hline
\end{tabular}

Fig. 1 Tableau synthétique des différentes stèles mises au jour en Île-de-France. Présentées par département et par ordre alphabétique.

Cette recherche a permis d'estimer un nombre minimal de 285 stèles mises au jour en Île-de-France dans 35 sites distincts. Parmi celles-ci, au moins 121 présentent un décor, connu pour 118 stèles (cf. fig. 1). La répartition des stèles funéraires ainsi obtenue révèle de fortes disparités locales (fig. 2). On constate ainsi une concentration importante de sites funéraires ayant livré des stèles dans l'Ouest parisien, avec 19 sites recensés dans le Val-d'Oise. Ils sont présentés ici dans l'ordre alphabétique. Des découvertes ont été répertoriées dans la nécropole des Buis à Ableiges ${ }^{19}$, mise au jour en 1887. À Amenucourt ${ }^{20}$, deux stèles ont été découvertes au Val Perron en 1956-1957 avec une quarantaine de sépultures, dont plusieurs inhumées en sarcophages de plâtre ou de pierre. À Baillet-en-France ${ }^{21}$, une stèle est apparue dans les labours à proximité d'un édifice religieux. D'autres ont été régulièrement mises au jour dans la nécropole de Banthelu à partir de $1957^{22}$. Une stèle a été mise au jour dans une nécropole fouillée dans les années 1970

19. Sirat 1966; Id. 1970; Sirat, Vieillard-Troїekouroff, Chatel 1984, p. 25-27, p. 64-67, p. 118-119, p. 303-307.

20. Sirat, Vieillard-Troї̈kouroff, Chatel 1984, p. 27-28; Wabont, Abert, Vermeersch 2006, p. 132.

21. GuAdagnin 1988, p. 171.

22. Sirat 1966; ID. 1970; ID. 1973; Sirat, Vieillard-Troïekouroff, Chatel 1984, n² 29-39. près de l'agglomération antique de Beaumont-sur-Oise ${ }^{23}$. À Condécourt ${ }^{24}$, des sondages explorant en 1980 la nécropole mérovingienne ont livré quelques stèles. Près de l'ancienne agglomération gallo-romaine de Genainville, deux stèles auraient été repérées au sein d'une nécropole au lieu-dit la Couture $^{25}$ et une autre sur le site monumental des Vaux-dela-Celle ${ }^{26}$. À Guiry-en-Vexin, quelques stèles mises au jour en 1958 proviennent de la nécropole de la Nourotte ${ }^{27}$ et une autre de celle explorée en 1954-1955 au lieu-dit Sur-les-Quais ${ }^{28}$. Des stèles ont été découvertes en 1963 dans une petite nécropole à Longuesse ${ }^{29}$. Une stèle mérovingienne a été identifiée en 1993 dans l'église Saint-Just à Louvres ${ }^{30}$. Quelques stèles proviennent du site funéraire de Blamecourt ${ }^{31}$, découvert en 1952 à Magny-en-Vexin. Une stèle a été recueillie avec

23. Wabont, Abert, Vermeersch 2006, p. 173-174.

24. Sirat, Vieillard-Troїekouroff, Chatel 1984, p. 17-18, et no 210.

25. Sirat 1966, p. 74-75; Mitard 1973; Sirat 1981; Sirat, Vieillard-TroḯkourofF, Chatel 1984, no $70 ; n^{\circ}$ 215-216.

26. MitARD 1973, p. 112.

27. Sirat 1966; Sirat, Vieillard-TroÏekouroff, Chatel 1984, nº 71.

28. Wabont, Abert, Vermeersch 2006, p. 297-298.

29. Sirat 1966; Sirat, Vieillard-Troї̈kouroff, Chatel 1984, nº 76-78.

30. Wabont, Abert, Vermeersch 2006, p. 319-320.

31. Sirat 1970; Sirat, Vieillard-Troïekouroff, Chatel 1984, nº 67-69. 


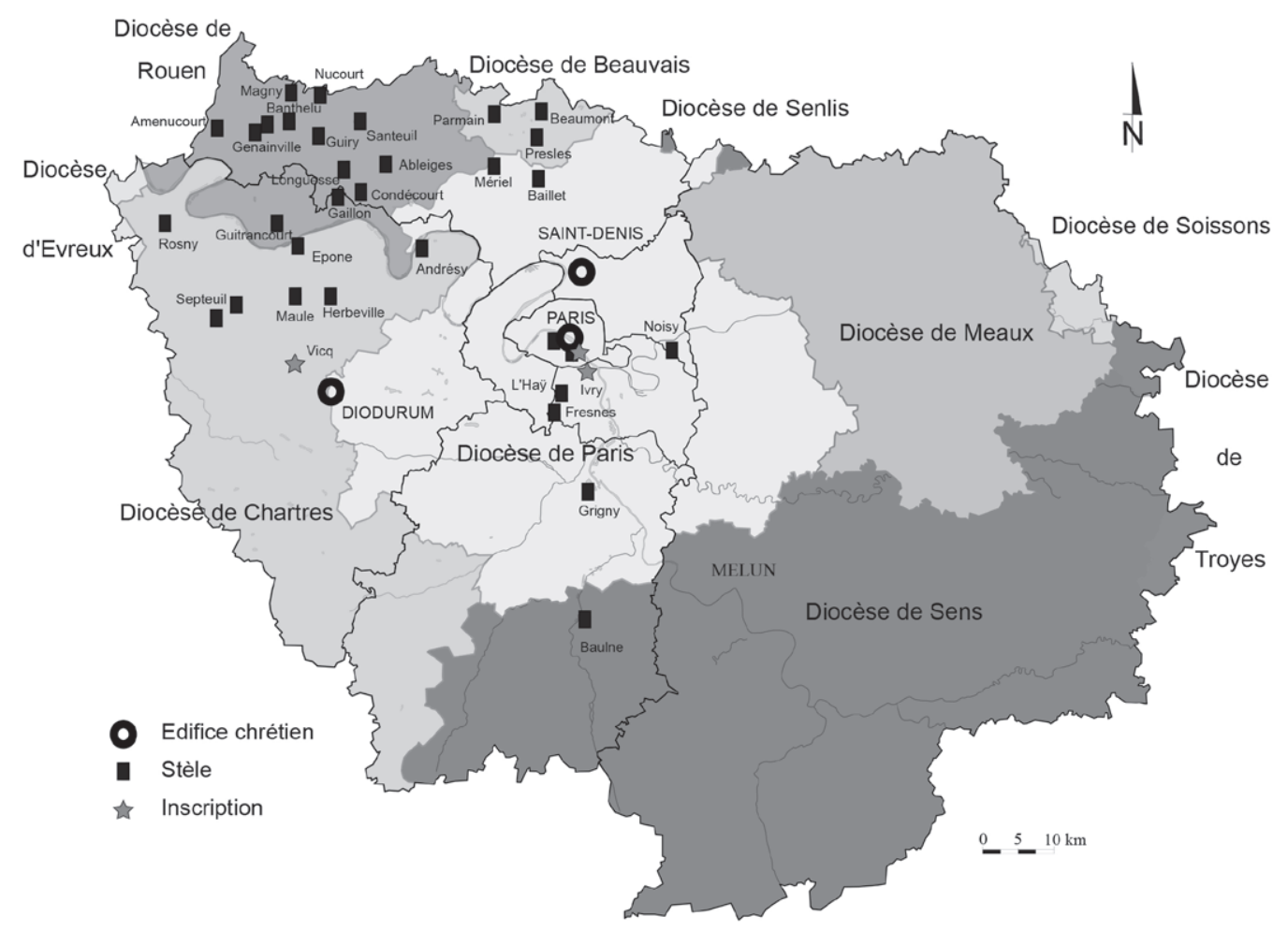

Fig. 2 Carte de localisation des stèles mérovingiennes en île-de-France (dessin S. Ardouin).

des sépultures mises au jour en 1972 lors de la construction $\mathrm{du}$ collège Claude-Monet, toujours à Magny-en-Vexin ${ }^{32}$. Également en Val-d'Oise, sept stèles auraient été mises au jour entre 1921 et 1924 parmi les sarcophages de la nécropole des Stors à Mériel ${ }^{33}$. À Nucourt ${ }^{34}$, deux stèles auraient été découvertes vers 1883. À Parmain ${ }^{35}$, une fouille menée en 1970-1971 en a livré trois dans une nécropole comprenant sarcophages de pierre et de plâtre. Une nécropole repérée en 1901 à Presles ${ }^{36}$, à la Côte-des-Trépassés, aurait livré quelques stèles ornées. À Santeuil enfin ${ }^{37}$, une importante nécropole fouillée en 1987-1988 en a également révélé plusieurs.

Toujours dans l'Ouest parisien, le département des Yvelines comporte neuf sites ayant fourni des stèles. La nécropole d'Andrésy, mise au jour en $1890^{38}$, en a livré plusieurs. Quelques-unes proviennent de la fouille menée en 1973 sur la nécropole d'Epône ${ }^{39}$. Celle de Gaillon-sur-Moncient ${ }^{40}$ explorée en 1996 en a également révélé. La nécropole de Guitrancourt ${ }^{41}$, mise au jour dans une carrière en 1953, en a livré d'autres. Une

32. Sirat, Vieillard-Troïekouroff, Chatel 1984, nº 80, p. 44.

33. Sirat 1980; Wabont, Abert, Vermeersch 2006, p. 345.

34. Sirat 1966; Sirat, Vieillard-Troïekouroff, Chatel 1984, nº 219.

35. Sirat, 1970 ; Sirat, Vieillard-Troïekouroff, Chatel 1984, nº 174-177.

36. Wabont, Abert, Vermeersch 2006, p. 381.

37. Sirat 1970; Sirat 1993; Sirat, Vieillard-Trö̈ekouroff, Chatel 1984, n 172, 220; MAZEAU 2006.

38. Sirat 1966; Sirat 1970; Sirat, Vieillard-Troïekouroff, Chatel 1984, n²0-27, p. 194-206.

39. Sirat, Vieillard-Troḯkouroff, Chatel 1984, n 300-302, p. 116-118. 40. RÉGNARD 1996; RÉGNARD 2001; RÉGNARD 2002; RÉGNARD, LANGLOIS, VANPEENE 2002, p. 71.

41. Sirat 1966; Sirat, Vieillard-TroÏekouroff, Chatel 1984, nº 241-244. stèle découverte fortuitement en 1965 au lieu-dit les Closeaux, à Herbeville, semble provenir d'un contexte plutôt antique ${ }^{42}$. Plusieurs stèles proviennent de la vaste nécropole de Maule ${ }^{43}$, fouillée entre 1954 et 1968 . Une seule a été mise au jour au sein de la nécropole découverte en 1892 autour de l'ancienne église Saint-Lubin de Rosny-sur-Seine ${ }^{44}$. À proximité de l'agglomération antique de Septeuil, une stèle isolée a été mise au jour près d'un sanctuaire de source aux Grands Bilheux ${ }^{45}$. Toujours à Septeuil, des stèles proviennent d'une nécropole fouillée au lieu-dit la Pierre Bât en $1984^{46}$.

Elles sont presque anecdotiques ailleurs. La nécropole des Mastraits à Noisy-le-Grand, seul site identifié en Seine-SaintDenis, a révélé 23 stèles ${ }^{47}$. Dans l'Essonne, seuls deux ensembles funéraires comportaient probablement des stèles funéraires. À Grigny ${ }^{48}$, au nord du département, quelques dalles portant des stries pourraient avoir eu cet usage. Plus au sud, à Baulne ${ }^{49}$, des stèles monolithiques ont pu marquer l'emplacement de sépultures. Dans le Val-de-Marne, la nécropole de la Tour aux Chartiers à Fresnes ${ }^{50}$ en a livré une en 1975. Celle mise au jour dans les carrières de L'Haÿ-les-Roses, sur les hauteurs en

42. BARAT 2007, p. 190-191, fig. 244.

43. Sirat 1973 ; Sirat, Vieillard-TroÏekouroff, Chatel 1984, nº 117-167.

44. Sirat, Vieillard-Troïekouroff, Chatel 1984, nº 259.

45. BARAT 2007, p. 335.

46. BARAT et al. 2001, p. 154-155; BARAt 2007, p. 338.

47. Site des Mastraits (Le Forestier 2015, p.201-202). Nous tenons particulièrement à remercier Cyrille Le Forestier pour nous avoir permis d'utiliser ses clichés photographiques.

48. NAudet 2004, p. 169.

49. Ibid., p. 109.

50. Boucher 1975; Ardouin 2006, p. 59-61. 


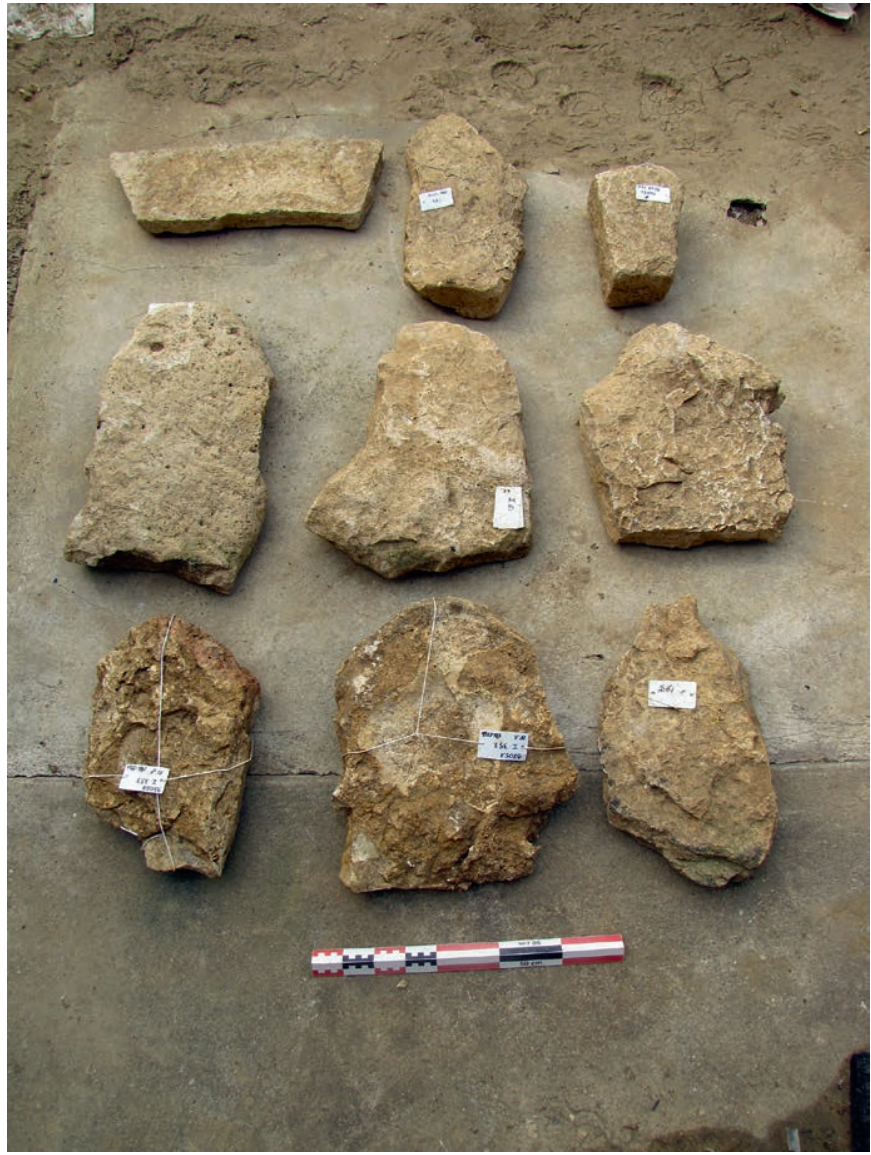

Fig. 3 Sélection de stèles mises au jour à Noisy-le-Grand (cl. C. Le Forestier, Inrap).

limite d'Arcueil-Cachan, en contenait avec certitude ${ }^{51}$. À Paris, seules deux stèles ont été identifiées ${ }^{52}$. Aucune n'a été identifiée dans les Hauts-de-Seine et, plus étonnamment, au regard de sa superficie, en Seine-et-Marne.

La rareté des stèles en dehors des zones nord-ouest de l'Îlede-France peut en partie s'expliquer par leur disparition au fil du temps. Pour autant, de telles différences ne peuvent être uniquement justifiées par les seules destructions. La présence d'inhumations ne se recoupant pas ou peu au sein de vastes nécropoles indique vraisemblablement une signalisation des sépultures en surface. L'examen plus attentif de la documentation concernant certains sites pourrait permettre de réévaluer cet inventaire, peut-être sous-estimé dans le sud et l'est. La mise au jour récente des 23 stèles dans la nécropole des Mastraits à Noisy-le-Grand, en Seine-Saint-Denis, a priori sans décors et identifiées par leurs formes ${ }^{53}$, pourrait indiquer que l'absence de motifs figurés sur les stèles du sud et de l'est franciliens pourrait avoir contribué à minorer le décompte des découvertes. Les stèles des Mastraits sont presque toutes en meulière (fig. 3), roche sédimentaire siliceuse présentant un aspect caverneux, à l'inverse de celles du Vexin, principalement taillées dans des calcaires fins à surfaces plus homogènes. Des stèles non

51. Giraud 1929; Ardouin 2006, p. 58.

52. Saint-Germain-des-Prés (PÉRIN [dir.] 1985, p. 205); Saint-Marcel (PÉRIN [dir.] 1985, p. 288).

53. Le Forestier 2015, p. 201-202.

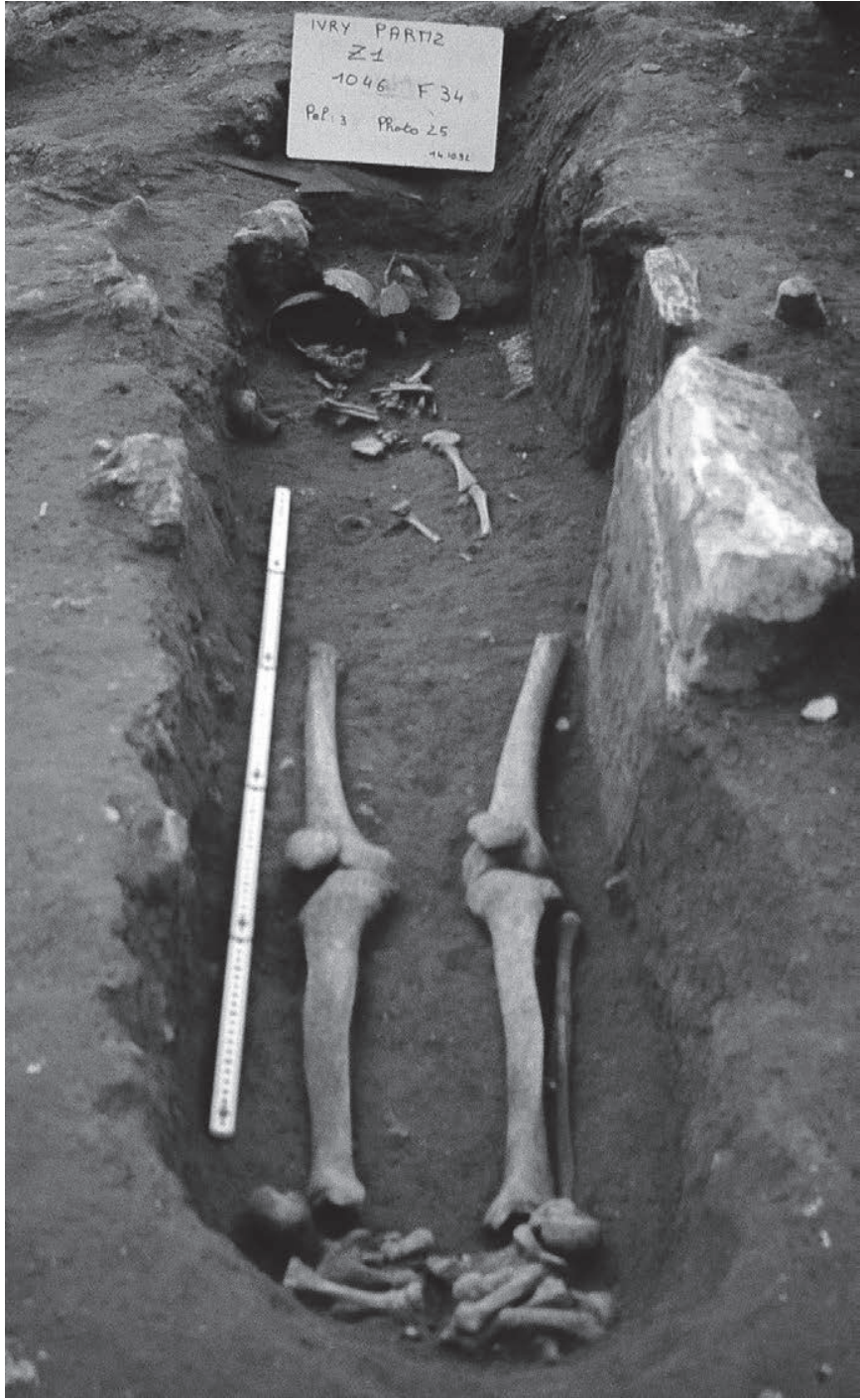

Fig. 4 Sépulture d'enfant ayant réemployé l'emplacement d'une sépulture d'adulte à Ivry-sur-Seine, près de la chapelle SaintFrambour (cl. B. Allard, Service départemental d'archéologie du Val-de-Marne).

décorées et brisées, sans forme particulière et sommairement taillées, ont pu ne pas être identifiées lors de fouilles ou découvertes fortuites anciennes. Certains aménagements mis au jour en 1992-1993 dans la nécropole d'Ivry-sur-Seine, près de l'ancienne chapelle Saint-Frambour ${ }^{54}$, pourraient avoir facilité le signalement des sépultures en surface, permettant la réutilisation de l'emplacement des sépultures. Une pierre calcaire était ainsi posée verticalement (remploi lapidaire antique supposé, non identifié comme stèle lors de la fouille) sur le côté d'une inhumation (fig. 4). Elle est probablement datable de la fin du VI ${ }^{e}$ ou du début du vire siècle (phase MA3-MR1) par une plaque-boucle à plaque ronde $\mathrm{e}^{55}$ mise au jour dans le comblement des sépultures qui ont remployé l'emplacement au viI ${ }^{e}$ siècle $^{56}$.

\section{Mousset 1999.}

55. Ardouin 2001, p. 47, fig. 7, n 1 (type 157 de Legoux, Périn, VAlet 2016). 56. L'une d'entre elles était accompagnée d'une fibule ronde décorée d'ocelles à umbo central : Ardouin 2001, p. 47, fig. 7, n 3 (type 219 de Legoux, Périn, VALET 2016). 
D'autres stèles ont donc pu ne pas être signalées ou conservées. Cette explication ne peut toutefois justifier l'absence de données sur l'ensemble des nécropoles du sud-est ${ }^{57}$. La présence de stèles en pierre pourrait donc révéler des usages locaux différents et notamment l'utilisation préférentielle de matériaux périssables tels que le bois hors de ce secteur. Des trous de poteau ont parfois été découverts à proximité des inhumations. Quelques rares trous de poteau jouxtaient ainsi des sépultures mises au jour au Tremblay-en-France (Seine-Saint-Denis) en 1997-1998 ${ }^{58}$, mais la relation entre les structures n'est pas assurée. Ces cas demeurent anecdotiques, des aménagements de ce type ayant probablement disparu car, sans doute moins profondément creusés que les sépultures.

Les stèles mérovingiennes, concentrées dans le Vexin français, pourraient-elles constituer une particularité de ce secteur du diocèse de Rouen, puisqu'on les rencontre également sur les limites avec les diocèses de Beauvais (Parmain), de Chartres (Rosny-sur-Seine et plus en retrait Maule et Septeuil) et de Paris (Mériel et Andrésy)? Elles sont cependant très présentes en dehors de la province ecclésiastique de Rouen, en Belgique seconde (surtout, si l'on prend le découpage administratif contemporain, en Picardie, dans les départements de l'Oise, la Somme et l'Aisne ${ }^{59}$ ) et plus sporadiquement au sud-est dans le diocèse de Paris (Paris, L'Haÿ-les-Roses, Fresnes, Noisy-leGrand, Grigny et probablement Baulne).

Le nombre de stèles varie d'une nécropole à l'autre. Sept sites ont livré plus de dix stèles (52 au maximum ${ }^{60}$ ) et cinq nécropoles comptabilisent entre cinq et neuf stèles ${ }^{61}$. Les 118 stèles recensées présentant un décor (autre qu'une simple cavité) correspondent généralement à plus de $50 \%$ des effectifs des sites les plus importants ${ }^{62}$.

Les stèles sont-elles représentatives des effectifs des ensembles funéraires? Selon notre estimation réalisée sur les nécropoles, même partiellement fouillées, présentant des effectifs de plus de 100 sépultures, le pourcentage de stèles varie d'un site à l'autre. Elles représentent au moins $3 \%$ des inhumations mises au jour à Andrésy, qui comporte au minimum 15 stèles pour 492 sépultures datées entre la fin du ve et le viII ${ }^{\mathrm{e}}$ siècle. Cette représentation peut monter à $9 \%$ dans la nécropole de Santeuil (17 stèles recensées pour au moins 193 sépultures entre le $\mathrm{v}^{\mathrm{e}}$ et le viII ${ }^{\text {e }}$ siècle) ou 10,5\% à Noisy-le-Grand (23 stèles parmi les 219 inhumations datées de la période $\mathrm{VI}^{\mathrm{e}}$-début $\mathrm{VIII}^{\mathrm{e}}$ siècles $\left.{ }^{63}\right)$. La proportion de stèles peut monter à 22-23\% à Gaillon-surMontcient (50 stèles ou fragments de stèles pour 220 sépultures datées entre la fin du $\mathrm{V}^{\mathrm{e}}$ et la deuxième moitié du VII ${ }^{\mathrm{e}}$ siècle) ou Banthelu (39 stèles pour 161 inhumations entre les $\mathrm{VI}^{\mathrm{e}}$ et $\mathrm{X}^{\mathrm{e}}$ siècles).

57. BARAT 2007, p. 363.

58. FrÈre, HÉron 1998

59. Flèche Mourgues 1998.

60. 52 stèles à Maule; 50 à Gaillon-sur-Montcient; 39 à Banthelu; 23 à Noisyle-Grand; 22 à Ableiges; 17 à Santeuil; 15 stèles au minimum à Andrésy.

61. 9 stèles à Epône; 7 à Mériel; 6 à Guitrancourt; 5 à Condecourt; 5 à Longuesse.

62. 19 stèles à Maule; 18 à Ableiges et Banthelu; 13 retenues à Gaillon-surMontcient; 12 à Santeuil; 9 à Andrésy.

63. Cette nécropole comportait également 409 inhumations carolingiennes (VIII ${ }^{\mathrm{e}}-\mathrm{XI}^{\mathrm{e}}$ siècles).

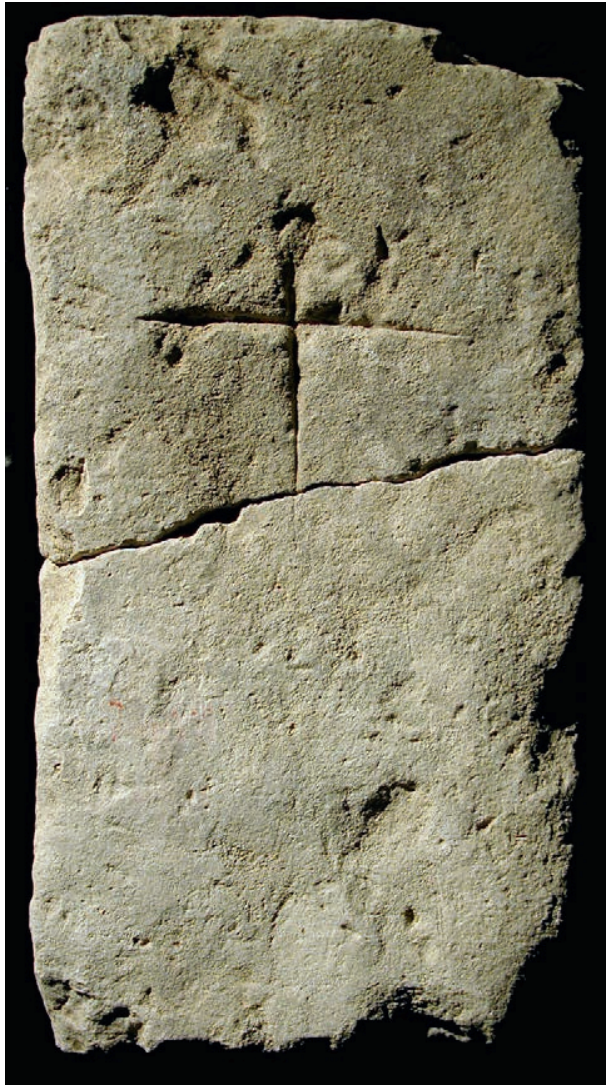

Fig. 5 Stèle découverte à Fresnes en couverture des sépultures 102 et 103 (cl. B. Allard, Service départemental d'archéologie du Val-deMarne).

\subsection{CONTEXTES DE DÉCOUVERTES}

Très peu de stèles semblent avoir été découvertes dans leur position initiale. Cela semble le cas sur quelques inhumations mises au jour en 1984 dans la nécropole de la Pierre Bât à Septeuil. Quelques pierres posées de chant à la tête d'un groupe de sépultures ( $\mathrm{n}^{\circ} 25,26$ et 32 ) ont pu jouer le rôle de stèles grossières. Deux pierres plantées de part et d'autre d'une sépulture voisine ( $\left.\mathrm{n}^{\circ} 34\right)$ auraient aussi pu avoir cette fonction $^{64}$. À Mériel, deux stèles auraient été découvertes posées verticalement sur les sépultures 10 et $11^{65}$. À L'Haÿles-Roses, deux stèles pourraient avoir été observées en place à la tête de deux sépultures ${ }^{66}$. Si certains signalements de découvertes associent les stèles aux sépultures, la plupart ne mentionnent pas l'emplacement de la stèle. De nombreuses stèles en place ont probablement été détruites par les labours, comme en témoignent de nombreuses découvertes dans ces conditions, à Magny-en-Vexin sur le site de Blamecourt en $1952^{67}$, à Baillet-en-France en $1967^{68}$, mais surtout à Banthelu

64. BARAT 2007, p. 338.

65. SIRAT 1980.

66. Giraud 1929; Ardouin 2006, p. 58, fig. 4.

67. Sirat 1970, p. 100; Sirat, Vieillard-TroÏekouroff, Chatel 1984, $\mathrm{n}^{\circ} 67,69$.

68. Guadagnin 1988, p. 171 ; Wabont, Abert, Vermeersch 2006, p. 151. 

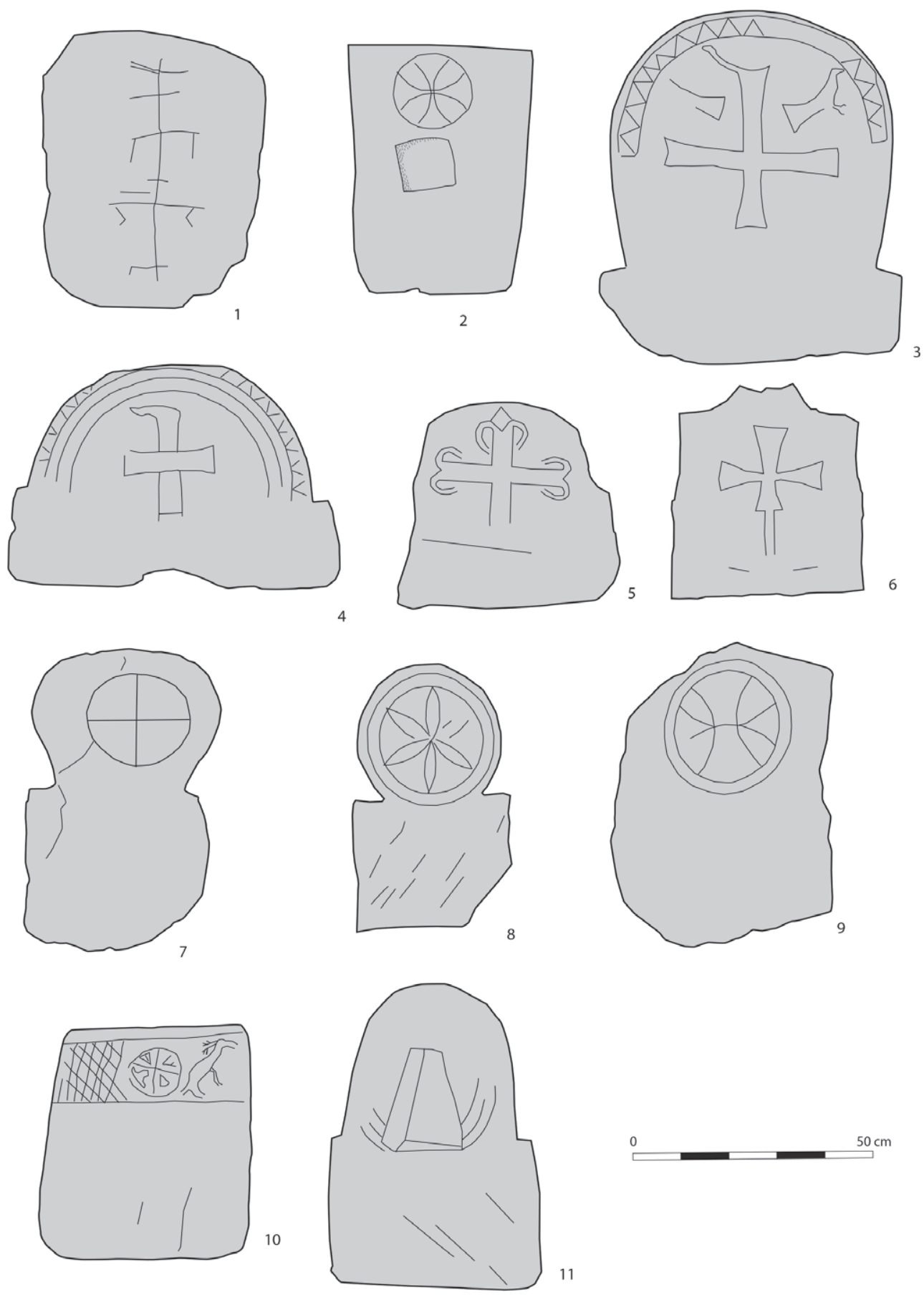

Fig. 6 № 1 Guitrancourt (sép. 45); № 2 Ableiges (sép. 19); № 3 Ableiges (sép. indéterminée); № 4 Ableiges (sép. 17); № 5 Santeuil (sép. 146); № 6 Gaillon-sur-Montcient (sép. 81); № 7 Gaillon-sur-Montcient (sép. 82); № 8 Maule (sép. 657); № 9 Maule (sép. indéterminée); № 10 Andrésy (sép. indéterminée); № 11 Maule (sép. 603) (DAO S. Ardouin : d'après photos [nº 1, 2, 3, 4, 5, 7, 8, 9], d'après SIRAT 1966 [nº 10], d'après RÉGNARD $\left.2001\left[n^{\circ} 6\right]\right)$.

en $1957^{69}, 1961^{70}$ et plusieurs fois encore après cette date ${ }^{71}$. À Banthelu toujours, une stèle mise au jour dans les labours était positionnée au-dessus d'une sépulture ( $\left.\mathrm{n}^{\circ} 51\right)$ fouillée par la

69. Sirat 1966, p. 76; Sirat, Vieillard-Troïekouroff, Chatel 1984, nº 29. 70. Sirat 1966, p. 78; Sirat, Vieillard-Trö̈ekouroff, Chatel 1984, nº 30. 71. Sirat 1970, p. 100, 102; Sirat, Vieillard-Troïekouroff, Chatel 1984 , $\mathrm{n}^{\circ} 52,53,56,59,61,64$. suite $^{72}$. C'est probablement le cas aussi d'une stèle semi-circulaire mise au jour en 1958 couchée sur une sépulture à Guiryen-Vexin/la Couture ${ }^{73}$.

72. Sirat 1970, p. 98; Sirat 1973, p. 117; Sirat, Vieillard-TroÏekouroff, Chatel 1984, no 48.

73. Wabont, Abert, Vermeersch 2006, p. 284. 
Les stèles sont principalement découvertes remployées, notamment en couverture. À Gaillon-sur-Montcient, si l'on excepte trois exemplaires mis au jour dans les déblais, toutes les stèles ont été remployées dont 34 en couverture ${ }^{74}$. Six sépultures parmi les 219 découvertes à Noisy-le-Grand comportaient des stèles remployées en couverture. Trois en comportaient plusieurs ${ }^{75}$. Plusieurs stèles peuvent être remployées en couverture d'une même sépulture, comme la sépulture $\mathrm{n}^{\circ} 74$ de Gaillon-sur-Montcient, couverte avec quatre stèles ${ }^{76}$. La réutilisation de stèles en couverture de sarcophages de plâtre a été observée à Santeuil (deux stèles pour la sépulture $\mathrm{n}^{\circ} 85^{77}$ ), à Fresnes (fig. 5 et 12, une stèle brisée répartie sur deux sépultures voisines $\mathrm{n}^{\circ} 102$ et $\mathrm{n}^{\circ} 103^{78}$ ), à Guitrancourt (une stèle sur la sépulture $\mathrm{n}^{\circ} 45^{79}$ : fig. $6, \mathrm{n}^{\circ} 1$ ) ou encore à Mériel (sépulture $\left.\mathrm{n}^{\circ} 9^{80}\right)$. Dans cette dernière nécropole, six autres stèles mises au jour renversées dans la tombe ou debout au milieu ou au pied du sarcophage ${ }^{81}$, pourraient correspondre aux couvertures effondrées. À Condecourt, quatre stèles ont été réutilisées en couvercle de sarcophages de plâtre ${ }^{82}$. Elles peuvent également couvrir des sarcophages de pierre, comme cela a été observé à Santeuil (sépulture $\mathrm{n}^{\circ} 28^{83}$ ) et Banthelu (sépultures n 147 et $155^{84}$ ), ou des demi-cuves de pierre comme, à nouveau, à Santeuil (sépultures no 112 et $113^{85}$, cf. fig. 13). Plusieurs stèles mises au jour dans les nécropoles d'Ableiges (fig. $6, \mathrm{n}^{\circ} 2$ et 4 ; fig. $8, n^{\circ} 5$ ) ou de Maule (fig. $6, n^{\circ} 8$ ), associées à des sépultures, proviennent vraisemblablement des couvertures. Dans certains cas (Santeuil, sépulture $\mathrm{n}^{\circ} 85$; Fresnes, sépultures $\mathrm{n}^{\circ} 102$ et 103), il semble que le sarcophage qui remploie des stèles en couverture soit lui-même réutilisé, avec réduction des sépultures d'origine, dont les ossements ont parfois été rassemblés, comme à Fresnes, au-dessus de la couverture.

D'autres stèles ont simplement été mises au jour dans le remblai de la fosse sépulcrale, comme à Santeuil (sépulture $n^{\circ} 135^{86}$ ) ou Guiry-en-Vexin la Couture ${ }^{87}$. À Santeuil également, une stèle ornée fut découverte dans le comblement de la sépulture $\mathrm{n}^{\circ} 146^{88}$ (fig. $6, \mathrm{n}^{\circ}$ 5), qui avait été bouleversée par un pillage.

Les stèles pouvaient également être placées verticalement, intégrées au coffrage de la sépulture pour servir de support à la couverture. C'est le cas d'une stèle posée à la tête de la

74. RÉGNARD 2001, p. 199.

75. Le Forestier 2015, p. 201-202.

76. RÉGNARD 2001, p. 92-93, pl. 60.

77. Mazeau 2006, p. 38-39, 56, 66, 85.

78. Boucher 1975; Ardouin 2006, p. 59-61, fig. 6.

79. Sirat 1966, p. 80 ; Sirat, Vieillard-Troïekouroff, Chatel 1984, $\mathrm{n}^{\circ} 241$

80. Sirat 1980; Wabont, Abert, Vermeersch 2006, p. 345.

81. Wabont, Abert, Vermeersch 2006, p. 345.

82. Ibid., p. 215.

83. Mazeau 2006, p. 31, 66, 86.

84. Sirat 1970, p. 97, 100, pl. I, fig. 59, 67; Sirat, Vieillard-TroÏekourofF, Chatel 1984, n $43,51$.

85. Mazeau 2006, p. 42-43, 58, 66, 85.

86. Ibid., p. 45-46, 66, 85.

87. Wabont, Abert, Vermeersch 2006, p. 284.

88. Mazeau 2006, p. 47, 59, 66, 86, 87.

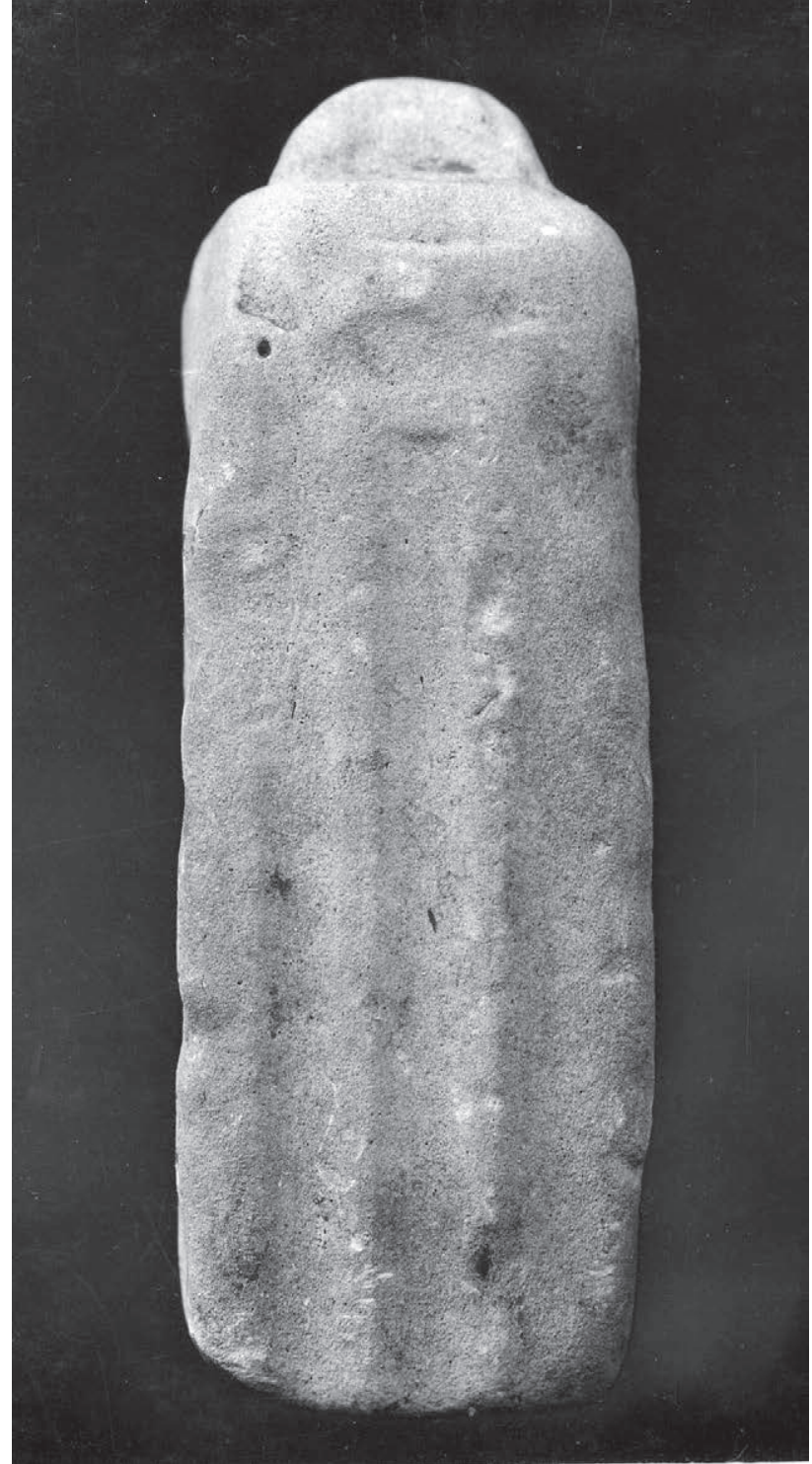

Fig. 7 Stèle mise au jour à L'Haÿ-les-Roses, sablière Gendre en 1927 (cl. anonyme. Documentation du Service départemental d'archéologie du Val-de-Marne).

sépulture $\mathrm{n}^{\circ} 81$ de Gaillon-sur-Montcient ${ }^{89}$ (fig. 6, $\mathrm{n}^{\circ} 6$ ), qui comportait également deux stèles en couverture, mais probablement également d'exemplaires mis au jour en bordure des sépultures $n^{\circ} 86$ et 90 de Banthelu ${ }^{90}$.

\subsection{FORMATS ET DÉCORS DES STÈLES}

En Île-de-France, les stèles sont essentiellement taillées dans des calcaires tendres, souvent des calcaires coquilliers, même si quelques exemplaires peuvent être extraits de calcaires plus grossiers (deux exemplaires sur 50 à Gaillon-sur-Mont-

89. RÉGNARD 2001, p. 99-100, pl. 62; RÉGNARD 2002, p. 44; RÉGNARD, LANGLOIS, VANPEENE 2002, p. 71.

90. Sirat 1970, p. 98, pl. I, fig. 65, 66; Sirat, Vieillard-Troïekouroff, Chatel 1984, n $49,50$. 
cient $^{91}$ ) ou en meulière (à Noisy-le-Grand ${ }^{92}$ ). Chaque stèle peut être classée selon la classification développée pour la Picardie par M.-P. Flèche Mourgues ${ }^{93}$. Les trois catégories typologiques principales sont les stèles simples, les stèles à sommet sur base débordante et les stèles à sommet fin et base épaisse. Les supports peuvent être de forme rectangulaire (fig. 5; fig. $6 \mathrm{n}^{\circ} 1,2,7,11$ ), trapézoïdale (fig. $6 \mathrm{n}^{\circ} 10$ ), à partie supérieure triangulaire (fig. $6 \mathrm{n}^{\circ} 6$ ), arrondie, à sommet discoïdale ou semi-circulaire (fig. $6 n^{\circ} 3,4,5,7,8$ ). De rares stèles présentent un sommet en acrotère (seuls cinq cas découverts, tous à Gaillon-sur-Montcient). Les tailles des stèles sont variables, mais se situent fréquemment entre 45 et $55 \mathrm{~cm}$ de haut pour $10 \mathrm{~cm}$ d'épaisseur, la largeur dépendant de la forme. Les plus grandes peuvent atteindre 70 à $80 \mathrm{~cm}$ de hauteur ou de largeur. Si la première stèle découverte à L'Haÿ-les-Roses en $1927^{94}$ est rectangulaire à sommet arrondi, la seconde est un petit monument original sans équivalent de $30 \mathrm{~cm}$ de hauteur, à sommet arrondi. Celle-ci présente un décor cannelé sur l'ensemble de sa base cubique, uniquement connu par une représentation photographique (fig. 7) et un descriptif sommaire, qui ne permettent pas d'estimer son volume réel originel ou l'éventualité d'un remploi de colonne antique.

Une stèle en cours de taille, mise au jour dans les ruines du sanctuaire antique de Genainville ${ }^{95}$ et destinée à être acheminée vers un autre lieu d'inhumation, indique que les stèles pouvaient faire l'objet d'une fabrication anticipée et non au coup par coup. Cette découverte pourrait révéler l'existence d'ateliers, expliquant certaines formes ou motifs récurrents sur plusieurs stèles malgré certaines divergences. Ces monuments pourraient donc faire l'objet d'un commerce, alors que l'importance du nombre de stèles et la variété des décors permettaient de supposer qu'elles pouvaient avoir été réalisées directement pour l'inhumation, au contraire des sarcophages ornés en pierre ou en plâtre dont le modèle pouvait être présumé de "série», réalisé avant son utilisation et stocké au préalable. La mise au jour de deux stèles à cavité de modules comparables (l'une est incomplète) découvertes à Santeuil, en couverture des sépultures $\mathrm{n}^{\circ} 106$ et $112^{96}$ présentant des décors identiques sur chaque face, à savoir une croix grecque d'un côté et une cavité sur l'autre, montre à l'évidence une reproduction à l'identique intentionnelle.

Des décors ont été observés sur les stèles provenant de nombreuses nécropoles. Si certains décors peuvent, comme pour les panneaux de sarcophages, être le résultat de compositions décoratives exécutées au compas (fig. 6, nº 8), d'autres révèlent des représentations d'inspiration chrétienne qui ne semblent guère contestables. C'est le cas de la croix monogrammatique de l'unique stèle de la nécropole de Fresnes ${ }^{97}$ (fig. 5 et fig. 8, $\mathrm{n}^{\circ} 4$ ), de la croix latine associée à la représentation schématique du christ (fig. 8, no 5$)^{98}$ de la nécropole d'Ableiges,

91. RÉGNARD 2001, p. 199.

92. Le Forestier 2015, p. 201-202.

93. Flèche Mourgues 1998.

94. Giraud 1929; Ardouin 2006, p. 58, fig. 4.

95. Mitard 1973, p. 112.

96. Mazeau 2006, p. 86, pl. 24.

97. Sépultures no 102 et 103 : Boucher 1975; Ardouin 2006, p. 59-61, fig. 6.

98. Sépulture no 14 : Sirat 1970, p.76, pl. I, fig. 18; Sirat, VieillardTroïekouroff, Chatel 1984, nº 19. d'une croix à crochet gravée sur deux stèles (fig. 6, n 3 et 4) de même provenance ${ }^{99}$. Ces deux croix à crochets pourraient d'ailleurs être des représentations de croix monogrammatiques latinisées, la boucle du rhô ayant été positionnée en sens contraire, l'inversion de caractères n'étant pas rares à la période mérovingienne ${ }^{100}$. L'une de ces croix à crochet est en effet accostée de deux oiseaux se tournant le dos (fig. 6, no 3 ), évoquant peut-être des colombes, fréquemment associées aux épitaphes chrétiennes, telle celle de Barbara (fig. 8, n 2) mise au jour à Paris dans la nécropole de Saint-Marcel ${ }^{101}$. Le caractère chrétien est manifeste également pour des stèles à croix fleuronnées, présentes uniquement à Santeuil ${ }^{102}$ (fig. 6, no 5 ) ou à croix ancrées, repérées à Banthelu ${ }^{103}$ et Santeuil ${ }^{104}$. Une dizaine de stèles à croix pattée identifiées sur des stèles d'Ableiges ${ }^{105}$, Gaillon-sur-Montcient ${ }^{106}$, Santeuil ${ }^{107}$ et Septeuil ${ }^{108}$ complètent cet ensemble. Il faut ajouter des croix pattées hampées ou processionnelles à Ableiges $^{109}$ et à Gaillon-sur-Montcient ${ }^{110}$ (fig. 6, no 6), une croix assez maladroite hampée et encerclée à Maule ${ }^{111}$, neuf exemplaires gravés de croix plus simples de type grec $^{112}$, et deux autres gravées de croix simples latines à

99. Sépultures no 17 et indéterminée: Sirat, Vieillard-TroÏEkouroff, Chatel 1984, no 15 et 18.

100. On peut citer les lettres apocalyptiques accompagnant un chrisme sur une stèle, mise au jour à Neuvion-en-Ponthieu dans la Somme (FLÈCHE Mourgues 1998, p. 107-108, fig. 4), dans le remblai d'une sépulture de la fin $\mathrm{du} \mathrm{v}^{\mathrm{e}}$ siècle ou du premier tiers du VI ${ }^{\mathrm{e}}$ siècle.

101. C.I.L., XIII, no 3052 (éd. Hirschfeld, Zangemeister 1899-1916); Duval 1960, t. 1, no 43, p. 106-108; Fossard, VieILlard-TroÏEKourofF, Chatel 1978, no 321, p. 165, pl. XCV.

102. Sépultures $\mathrm{n}^{\circ} 85,112,135,146$ et indéterminée: Sirat 1993, p. 221 ; MAzEAu 2006, p. 38-39, 56, 66, 85, 87 .

103. Sépultures $\mathrm{n}^{\circ} 86$ et indéterminée : Sirat 1970, p. 98 et 100, pl. I, fig. 65 et 68; Sirat, Vieillard-Troïekouroff, Chatel 1984, nº 49 et 52.

104. Sépulture no 28 : MAzEAU 2006, p. 31, 66, 85.

105. Sépultures $n^{\circ} 1$ et indéterminée: SirAt 1966, p. 78, pl. II, fig. 23, 24; Sirat, Vieillard-Troïekouroff, Chatel 1984, nº 183, 185.

106. Sépultures no 79, 80, 178 : RÉGNARD 1996, p. 102-103; RÉGNARD 2001, p. 96-99, 155-156, pl. 62 et 65; RÉGNARD 2002, p. 44.

107. Sépultures no 24 et 113 : MAzEAu 2006, p. 31, 42-43, 58, 66, 85.

108. Sépulture indéterminée: BARAT et al. 2001, p. 154-155, fig. 15; BARAT 2007, p. 338, fig. 547.

109. Sépulture no 8 : Sirat 1966, p. 78, pl. II, fig. 25; Sirat, VieillardTroïekouroff, Chatel 1984, n $184,184,185$.

110. Sépulture $\mathrm{n}^{\circ} 81$ avec une croix grossière sur la tranche supérieure: RÉGNARD 2001, p. 99-100, pl. 62 ; RÉGNARD 2002, p. 44; RÉGNARD, LANGLOIS, VANPEENE 2002, p. 71.

111. Sépulture indéterminée: Sirat, Vieillard-TroÏ̈kouroff, Chatel $1984, \mathrm{n}^{\circ} 134$.

112. Sépulture $\mathrm{n}^{\circ} 1$ d'Ableiges (Sirat 1966, p. 74, pl. I, fig. 20; Sirat, Vieillard-Troïekouroff, Chatel 1984, n 180); sépulture no 147 de Banthelu (Sirat 1970, p. 100, pl. I, fig. 67; Sirat, Vieillard-Trö̈̈KourofF, Chatel 1984, n० 51 ); sépulture $\mathrm{n}^{\circ} 3$ de Longuesse, la stèle présentant également une croix latine sur l'autre face (SIRAT 1966, p. 75, pl. I, fig. 13; Sirat, Vieillard-Troïekouroff, Chatel 1984, no76); sépultures 106 et 112 de Santeuil (MAzEAu 2006, p. 42, 58, 66, 86, 87); Sépulture indéterminée d'Andresy (Sirat 1970, p. 100, pl. II, fig. 71; Sirat, VieillardTroïenouroff, Chatel 1984, n² 24); sépulture n 178 de Gaillon-sur-Montcient (RÉGNARD 2001, p. 155-156, pl. 65); sépulture n 2 de Guitrancourt (Sirat, Vieillard-Troïekouroff, Chatel 1984, n²43); sépulture no 359 de Maule (Sirat, Vieillard-Troïekouroff, Chatel 1984, nº136). 

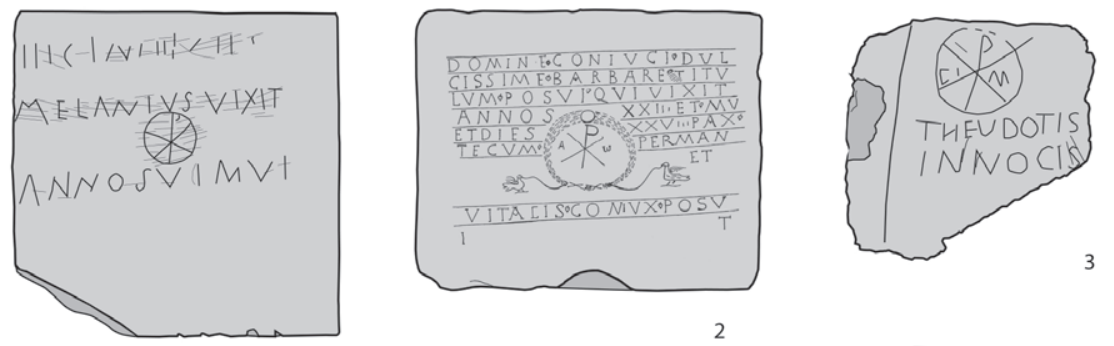

1

2
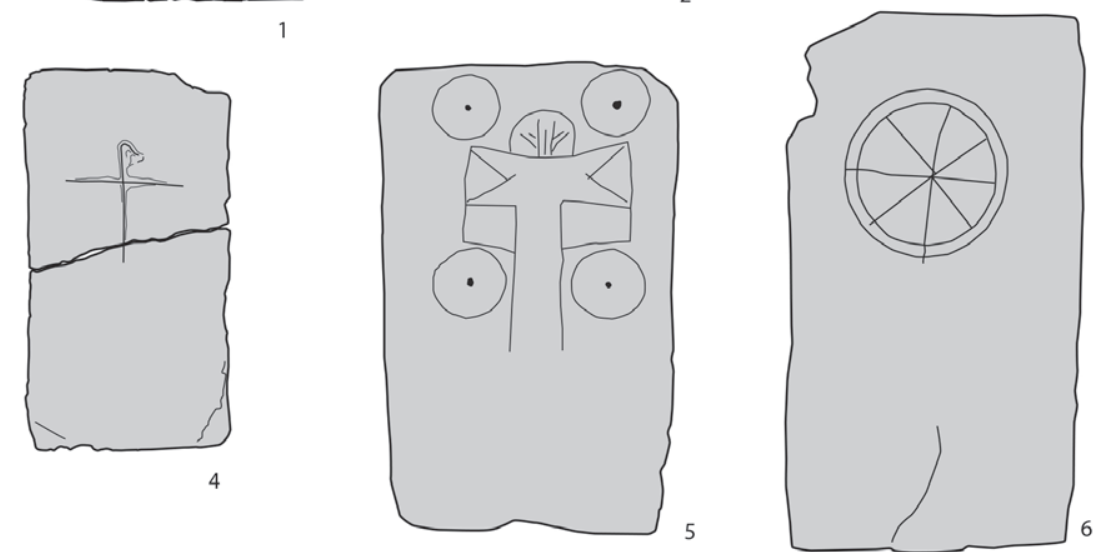
$50 \mathrm{~cm}$

Fig. 8 № 1 épitaphe d'Ivry-Saint-Frambourg (Melanius); n² épitaphe de Paris Saint-Marcel (Barbara); $n^{\circ} 3$ épitaphe de Paris Saint-Marcel (Theudote); $n^{\circ} 4$ stèle de Fresnes (sép. 102 et 103), no 5 stèle d'Ableiges (sép. 14); no 6 stèle de Magny-en-Vexin / Blamecourt (labours) (dessin S. Ardouin d'après l'original [no 1], d'après Duval 1960 [n' 2], dessins d'après photo [no 3, 4, 5 et 6]).

Banthelu ${ }^{113}$ et Maule ${ }^{114}$. Quelques motifs sont originaux, telle une croix latine gravée sur une stèle d'Andrésy, comportant une barre horizontale qui évoque une croix pattée et un cercle n'entourant que le centre de la croix ${ }^{115}$ (fig. 9, no 1 ).

Un exemplaire provenant de la nécropole d'Andresy ${ }^{116}$ comportait une croix pattée gemmée accostée de deux oiseaux. Une représentation de bouquetins accoste une autre croix gemmée sur une deuxième stèle provenant de cette nécropole ${ }^{117}$. Le caractère unique de ce type de croix accosté d'animaux semble indiquer qu'il pourrait s'agir d'une interprétation différente du décor d'une seule et même stèle (fig. $9 \mathrm{n}^{\circ} 2$ et 3 ). Une variation dans les relevés (la stèle ayant été retournée) est ainsi à l'origine d'une méprise aboutissant à la création de deux occurrences pour une seule et même stèle ${ }^{118}$ mise au jour dans cette même nécropole d'Andrésy (fig. 10, no 1, 2, 3). Pour les deux stèles à croix gemmée, on ne peut totalement écarter deux réalisations contemporaines dues à un même graveur. Ce type de décor peut

113. Sépulture no 51 (SIRAT 1970, p. 98, pl. I, fig. 64; SirAT 1973, p. 117, fig. 7 ; Sirat, Vieillard-Troïekouroff, Chatel 1984, nº 48).

114. Sépulture indéterminée (Sirat, Vieillard-TroÏ̈kouroff, Chatel 1984, $\left.\mathrm{n}^{\circ} 137\right)$.

115. Sirat 1966, p. 79, pl. II, fig. 29; Sirat, Vieillard-TroÏekouroff, Chatel 1984, n’22.

116. Sépulture indéterminée (Sirat 1966, p. 79, pl. II, fig. 32).

117. Sépulture indéterminée (Sirat, Vieillard-Trö̈̈кouroff, Сhatel 1984, n²07).

118. Sirat 1966, p. 79, pl. II, fig. 28 (stèle considérée comme perdue); Sirat 1970, p. 100, pl. II, fig. 74; deux occurrences créées dans Sirat, Vieillard-Troïekouroff, Chatel 1984, nº 25 et nº 204 (perdue). Cette stèle est conservée au Musée archéologique de Guiry-en-Vexin, inv. n M. 623.

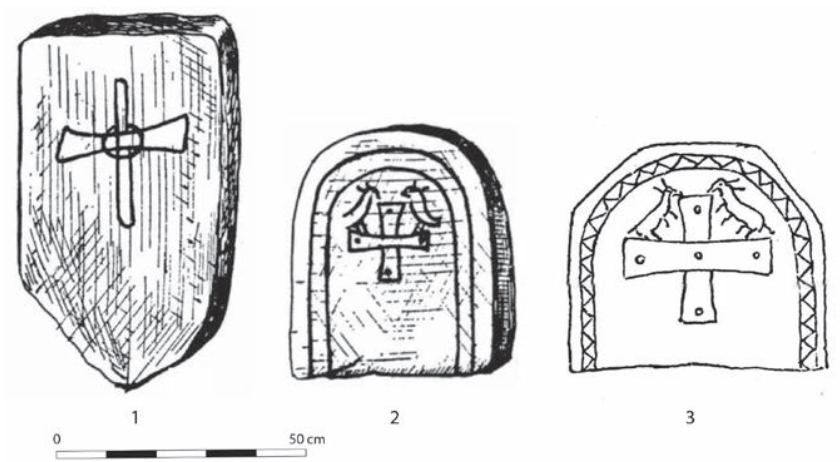

Fig. 9 Stèles d'Andrésy (sépultures indéterminées). № 1 d'après SIRAT 1966, pl. II, fig. 29. № 2 d'après SIRAT 1966, pl. II, fig. 32. № 3 d'après Sirat, Vieillard-Troiekouroff, Chatel 1984, n 207 (stèle perdue, échelle approximative).
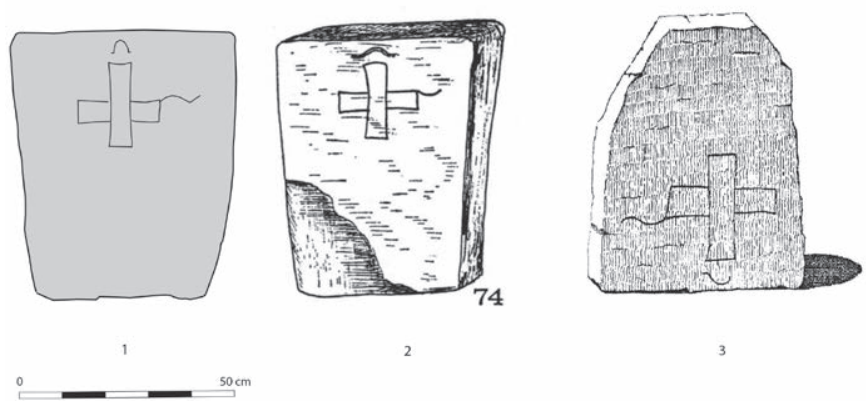

Fig. 10 Relevé d'une stèle d'Andrésy (sép. indéterminée). № 1 DAO S. Ardouin d'après photo. № 2 d'après SIRAT 1970, pl. II, fig. 74. № 3 d'après Sirat, Vieillard-Troiekouroff, Chatel 1984, no 204. 


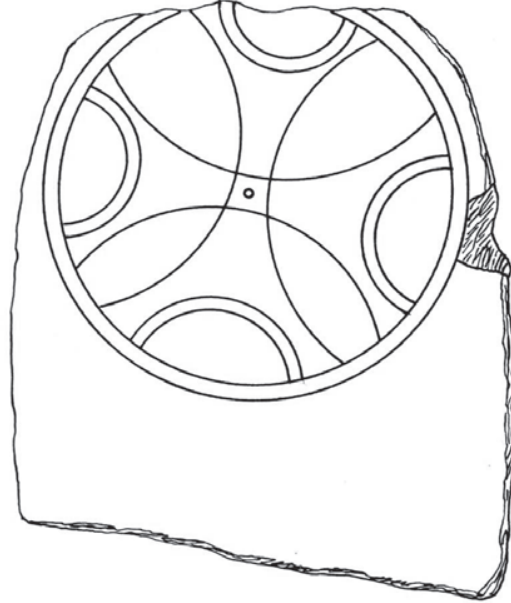

Fig. 11 Stèle d'Epône (d'après Sirat, Vieillard-TroieKouroff, Chatel 1984, $n^{\circ}$ 301) (dimensions indéterminées).

être rapproché d'une autre stèle d'Andrésy ${ }^{119}$ montrant un animal (probablement un cervidé) accosté d'une croix encerclée (fig. 6, $\left.\mathrm{n}^{\circ} 10\right)$. E. Salin estimait qu'il pouvait s'agir d'une évolution de motifs chrétiens. Les représentations animales étant rares et les relevés incertains, il est cependant difficile de se prononcer avec certitude sur l'intention des graveurs.

Au moins huit stèles réparties sur sept sites présentent des motifs que J. Sirat qualifiait de croix de Malte. Ce sont des stèles provenant des sites d'Ableiges ${ }^{120}$ (fig. 6, $\mathrm{n}^{\circ}$ 2), Banthelu ${ }^{121}$, Épône ${ }^{122}$, Maule ${ }^{123}$ (fig. 6, no 9), à Saint-Vincent de Paris ${ }^{124}$ (SaintGermain-des-Prés), Presles ${ }^{125}$ (dont le détail des gravures n'est pas connu) et Santeuil ${ }^{126}$. Ces croix de Malte encerclées sont les seuls motifs représentés sur ces stèles. Sur la stèle d'Épône (fig. 11), l'élaboration de la "croix» a été plus approfondie sur les extrémités des branches également tracées au compas. Pour ces représentations très géométriques, la désignation même de croix n'est pas évidente, ces motifs se rapprochant des rosaces réalisées au compas. Les supports de ces stèles ne sont pas exclusivement des dalles à partie supérieure arrondie ou à sommet discoïdale et sont fréquemment rectangulaires ou trapézoïdales, comme pour les autres motifs.

Les motifs parfois décrits comme des "chrismes simplifiés» ou « déformés ${ }^{127}$ », composés de croix grecques entrecoupées de

119. Sépulture indéterminée (SAlin 1952, p. 154, fig. 87; Sirat 1966, p. 82, pl. II, fig. 44; Sirat, Vieillard-TroÏekouroff, Chatel 1984, nº 199).

120. Sépultures no 16 et 19 (SIRAt 1966, p. 78, pl. I, fig. 21; pl. II, fig. 26; Sirat, Vieillard-Troïekouroff, Chatel 1984, nº 181 et 186.

121. Sépulture indéterminée (Sirat 1966, p.78, pl. I, fig. 19; Sirat, Vieillard-Trö̈eKouroff, Chatel 1984, n 30).

122. Sépulture indéterminée (Sirat, Vieillard-Troïekouroff, Chatel 1984, n 301).

123. Sépulture indéterminée (Sirat, Vieillard-Troïekouroff, Chatel 1984, no 138).

124. Sépulture indéterminée (PÉRIN (dir.) 1985, nº 144, p. 288.

125. Sépulture indéterminée (WABONT, ABERT, VERMEERsch 2006, p. 381).

126. Sépulture indéterminée (Sirat 1970, p. 100, pl. II, fig. 73; Sirat, Vieillard-Troïekouroff, Chatel 1984, n 172).

127. Sirat 1970, p. 98, 100; Sirat, Vieillard-TroÏekouroff, Chatel 1984, n 50 et 67 (chrisme simplifié); 122 et 139 (chrisme déformé). croix de Saint-André, ou de croix de Saint-André recoupées, sont représentés sur sept stèles découvertes à Saint-Marcel de Paris ${ }^{128}$, Banthelu ${ }^{129}$, Magny-en-Vexin ${ }^{130}$ (fig. 8, n 6) et Maule ${ }^{131}$. Leur comparaison avec les chrismes constantiniens tels qu'ils sont représentés sur les trois épitaphes mises au jour à Saint-Marcel de Paris ou à Saint-Frambour d'Ivry-sur-Seine paraît difficile, tant les représentations paraissent maladroites, contrastant avec la qualité de certaines figurations. On peut également interpréter ces pseudo-chrismes comme des motifs géométriques rayonnants encerclés, comparables aux rosaces représentées sur certaines stèles (fig. 6, nº 8).

En dehors des stèles figurées antiques et des motifs clairement ou susceptibles d'être attribuables au christianisme, l'analyse des représentations figurées demeure difficile, notamment pour certains motifs énigmatiques. Des signes indéchiffrables, difficiles à interpréter, composés de lignes entrecroisées, ont été notés sur plusieurs stèles anciennement découvertes à Ableiges ${ }^{132}$, Guitrancourt ${ }^{133}$ (fig. 6, no 1), Longuesse ${ }^{134}$ ou Herbeville ${ }^{135}$. Les cavités présentes sur la face de nombreuses stèles mérovingiennes du Vexin (fig. 6, no 11 , fig. 8, no 6) ont donné lieu à des interprétations différentes mais aucune ne paraît satisfaisante. L'hypothèse d'une évolution des stèlesmaisons gallo-romaines a été proposée puis écartée, celle d'une influence copte a été évoquée, par comparaison avec des stèles gréco-égyptiennes des $\mathrm{VI}^{\mathrm{e}}$-VII ${ }^{\mathrm{e}}$ siècles $^{136}$. La cavité était alors interprétée comme le réceptacle destiné à accueillir des reliques ${ }^{137}$. Si cette cavité put avoir une nécessité fonctionnelle, rien ne permet de justifier des influences coptes isolées dans ce secteur des Gaules. En l'absence d'explication satisfaisante, certains auteurs considèrent aujourd'hui qu'elles pourraient symboliser la dernière trace d'un paganisme local oublié, s'exprimant par ces pratiques funéraires particulières ${ }^{138}$. L'absence de précédents antiques ou de comparaisons germaniques ne permet pas d'étayer cette hypothèse.

128. Sépulture indéterminée (PÉRIN (dir.) 1985, nº 67 bis, p. 205).

129. Sépultures no 90 et 155 (Sirat 1970, p. 97, pl. I, fig. 59, 66; Sirat, Vieillard-Troïekouroff, Chatel 1984, no 43, 50).

130. Sépulture indéterminée de Blamecourt (SirAt 1970, p. 100, pl. II, fig. 69; Sirat, Vieillard-Troïekouroff, Chatel 1984, n 67).

131. Sépultures n 446 , et indéterminées (Sirat, VieILlard-TroÏEkourofF, Chatel 1984, no $122,139,162)$.

132. Sépulture no 3 (Sirat 1966, p. 82, pl. II, fig. 43; Sirat, VieillardTroïekouroff, Chatel 1984, no 192).

133. Sépulture $\mathrm{n}^{\circ} 3$ et 45 (Sirat 1966, p. 80, pl. II, fig. 40 et 39; Sirat, Vieillard-TroÏEkouroff, Chatel 1984, n 242 et 241, numérotée 238 par erreur) 134. Sépulture 13 (Sirat 1966, p. 82, pl. II, fig. 41; Sirat, VieillardTroïekouroff, Chatel 1984, nº 78)

135. Stèle isolée (BARAT 2007, p. 304-305).

136. L'origine de cette comparaison pourrait être SALIN 1952, p. 76.

137. Sirat 1970, p.74; Sirat 1973, p. 116; Sirat, Vieillard-TroiekouroufF, Chatel 1984, p. 16.

138. RÉGNARD 2002, p. 59. 


\section{CHRONOLOGIE DES STÈLES}

\subsection{LES STÈLES ANTÉRIEURES AU IVe SIÈCLE}

Des stèles monumentales ont été utilisées durant toute l'Antiquité. Elles pouvaient être inscrites, gravées de représentations divines ou présenter un personnage sculpté en bas-relief symbolisant le défunt. Les nécropoles urbaines ou périurbaines de Paris, Melun ou Meaux en ont livré plusieurs exemplaires ${ }^{139}$. L'une anépigraphe (cavalier romain terrassant un ennemi ${ }^{140}$ ), découverte en 1868 dans la nécropole parisienne de SaintMarcel, attribuée dans un premier temps au IV ${ }^{e}$ siècle, daterait du Haut-Empire ${ }^{141}$. Elles sont beaucoup moins connues en zone rurale. Une stèle gravée d'un personnage provient de Saint-Germain-lès-Arpajon ${ }^{142}$ (Essonne). Deux autres du même type sont connues à Avon ${ }^{143}$ (Seine-et-Marne). Deux fragments du $\mathrm{II}^{\mathrm{e}}$ siècle ont été découverts dans la nécropole de l'agglomération secondaire d'Épiais-Rhus ${ }^{144}$. Deux stèles du III siècle à représentation humaine grossière, découvertes à Saint-Sauveur-lès-Bray (Seine-et-Marne) ${ }^{145}$, semblent plus certainement provenir d'un laraire que de structures funéraires ${ }^{146}$. En Île-de-France, les stèles funéraires antiques demeurent donc une pratique essentiellement urbaine.

\subsection{DU IVe AU DÉBUT DU VIIII SIÈCLE}

La découverte d'une stèle en cours de taille dans les ruines du sanctuaire antique de Genainville ${ }^{147}$ permet d'indiquer que la fabrication de stèles put commencer tôt après le IV siècle, dès la mise en carrière des sites monumentaux antiques. Nous n'avons cependant que peu d'indices pouvant indiquer la fabrication de stèles avant le début du $\mathrm{VI}^{\mathrm{e}}$ siècle, période durant laquelle seules des épitaphes sont attestées, concentrées dans des nécropoles périurbaines autour de Paris (SaintMarcel ${ }^{148}$ et, un peu à l'écart, Ivry-sur-Seine ${ }^{149}$ ) ou de l'ancienne agglomération secondaire antique de Diodurum (JouarsPontchartrain) (nécropole de Vicq ${ }^{150}$ ).

Parmi les monuments les plus anciens pourrait figurer la stèle rectangulaire brisée, gravée d'une croix monogrammatique latinisée, le «chi» du monogramme ayant basculé pour former une croix latine, un « $\mathrm{R}$ » complétant l'hampe verticale en lieu et place de la boucle du «rhô» (fig. 5 et $8, n^{\circ} 4$ ). Elle a été découverte en 1974-1975 à Fresnes, en couverture de deux sarcophages voisins réutilisés, pour le plus ancien, entre 668

139. Cf. Busson 1998; Benhadou (dir.) 1990; Delattre, Magnan 1998; Griffisch, Magnan, Mordant 2008.

140. PACHTÈre 1912, p. 165

141. Duval 1961, p. 254 ; Busson 1998, p. 316.

142. NAUdeT 2004, p. 225, fig. 120.

143. Griffisch, Magnan, Mordant 2008, p. 245.

144. Wabont, Abert, Vermeersch 2006, p. 246.

145. Augereau 1995, p.79; Griffisch, Magnan, Mordant 2008, p. 1027-1029.

146. SÉGUiER et al. 2009.

147. Mitard 1973, p. 112.

148. Cf. Piétri 1985; Busson 1998; Ardouin 2006.

149. Ardouin 2001.

150. Le BLANT 1856, p. 301-302, n 210. et $802^{151}$. La nécropole qui pourrait avoir été en usage dès le IV ${ }^{e}$ siècle, semble régulièrement utilisée à partir de la fin du $\mathrm{V}^{\mathrm{e}}$ ou du VI $\mathrm{I}^{\mathrm{e}}$ siècle et durant tout le haut Moyen Âge, selon les analyses ${ }^{14} \mathrm{C}$, les contenants (sarcophages de pierre ou de plâtre ornés) et le mobilier métallique associé. Si l'on se fonde sur la typologie établie en Picardie, la forme rectangulaire serait tardive, postérieure à $575^{152}$. La croix monogrammatique, fréquente sur des épitaphes chrétiennes du ve siècle, ne se rencontre plus qu'exceptionnellement à la fin du VI ${ }^{e}$ siècle ${ }^{153}$. Ce motif est absent des stèles picardes ou vexinoises, alors que toutes les variétés de croix (latine, grecque, pattée, ancrée, etc.) y sont répertoriées. Cela accréditerait l'archaïsme de cette représentation, présente au $\mathrm{V}^{\mathrm{e}}$ siècle sur un tambour de colonne réutilisé en couvercle de sarcophage d'enfant à Paris, dans la nécropole de Saint-Marcel ${ }^{154}$. La stèle de Fresnes pourrait dater de la fin du $\mathrm{V}^{\mathrm{e}}$ ou du début du vie siècle, ce qui serait comparable à la datation des modules rectangulaires les plus anciens de Picardie.

À l'exception de la rare croix monogrammatique de Fresnes, la typologie des décors ne permet pas réellement d'affiner la datation des stèles. Pour les motifs rayonnants encerclés ou non, le rapprochement avec le chrisme est contestable dans la plupart des cas. Tombé en désuétude dès le $\mathrm{v}^{\mathrm{e}}$ siècle sur les épitaphes (la dernière inscription datée comportant ce décor est de 493), il n'apparaît plus que sporadiquement à Trèves jusqu'au $\mathrm{VII}^{\mathrm{e}}$ siècle ${ }^{155}$. Absent des monnayages mérovingiens des $\mathrm{VI}^{\mathrm{e}}$-VII ${ }^{\mathrm{e}}$ siècles, le motif du chrisme constantinien est relativement peu fréquent sur les panneaux de sarcophage de plâtre contemporains et dans ce cas souvent identifiable ${ }^{156}$. Si la volonté du graveur d'utiliser ce motif pour évoquer le monogramme du Christ ne peut être totalement écartée ${ }^{157}$, cette identification paraît cependant difficile à maintenir. Sur cinq exemplaires de stèles, le "chrisme» est au minimum doublement encerclé et donc très proche des rayons internes des décors dits «de rouelles» ornés de rosaces encerclées ou autres motifs rayonnants, parfois même composés uniquement de cercles successifs sans motif central. Le symbolisme chrétien ne semble pas indiscutable et ce motif ne peut donc être utilisé pour estimer l'ancienneté de ces stèles.

La datation des stèles par le contexte funéraire parait plus pertinente, même si peu de ces monuments semblent avoir été découverts dans leur contexte d'origine et plus souvent en remplois sur des inhumations postérieures.

Les datations avancées pour les fouilles anciennes, réactualisées, montrent que la nécropole d'Andrésy semble avoir été utilisée à partir de la fin du v ${ }^{e}$ ou du début du VI ${ }^{e}$ siècle

151. Datation ${ }^{14} \mathrm{C}$ de la tombe 102 : Ly-4577(GrA). Datation très fiable. 152. Flèche Mourgues 1992, p. 51 et tableau IV; Flèche Mourgues 1998, p. 113-114.

153. Gauthier 1975, p. 55-56; Descombes 1985, p. 76-77.

154. Busson 1998, p. 347-348.

155. Gauthier 1975, p. 55.

156. PÉRIN (dir.) 1985 : Saint-Marcel, n 86 p. 212 (identification contestable); Saint-Germain-des-Prés, n 201, 202, 203, 204, 205 p. 312-315.

157. La représentation de chrismes incontestables est aussi connue sur des stèles, notamment sur celle déjà évoquée de Neuvion-en-Ponthieu dans la Somme (Flèche Mourgues 1998, p. 107-108, fig. 4). Cette stèle, probablement en position secondaire, pourrait dater de la fin du viècle. 
jusqu'au viII siècle $^{158}$. D’après le mobilier répertorié, celle de L'Haÿ-les-Roses serait en activité aux VI ${ }^{\mathrm{e}}$-VII ${ }^{\mathrm{e}}$ siècles ${ }^{159}$. Les mêmes datations sont avancées pour Guitrancourt ${ }^{160}$. Bien que celle de Maule soit utilisée jusqu'au viri ${ }^{e}$ siècle, il semble que les stèles ne soient remployées que dans la phase antérieure, aux $\mathrm{VI}^{\mathrm{e}}$-VII ${ }^{\mathrm{e}}$ siècles $^{161}$. Les stèles de Banthelu seraient bûchées et réutilisées en couvercles de sarcophages dès le viI ${ }^{e}$ siècle ${ }^{162}$. À Septeuil, les sépultures les plus anciennes seraient du vi ${ }^{e}$ siècle et l'utilisation du cimetière se poursuivrait au viI ${ }^{\mathrm{e}}$ siècle ${ }^{163}$. La succession dans le temps des nécropoles de Guiry-en-Vexin semble nous apporter un élément supplémentaire : la Nourotte serait datée du $\mathrm{VI}^{\mathrm{e}}$ siècle et serait remplacée au VII ${ }^{\mathrm{e}}$ par celle découverte au lieu-dit Sur-les-Quais ${ }^{164}$. Ces données ont pu être complétées grâce à des fouilles plus récentes. À Gaillonsur-Montcient, les premières inhumations sont datées de la fin $\mathrm{du} \mathrm{v}^{\mathrm{e}}$ siècle et les stèles sont systématiquement détruites et réutilisées dans les dernières inhumations ${ }^{165}$, au cours de la seconde moitié du viI siècle. La sépulture no 79 (cf. fig. 14) est ainsi datée de la deuxième moitié du viI siècle par deux fibules ansées symétriques. La sépulture no 80 comportait le dépôt d'un vase biconique de la seconde moitié du viI ${ }^{\text {e }}$ siècle ${ }^{166}$. À Santeuil, les inhumations sont datées de la fin du ve siècle et se poursuivent jusqu'à la période carolingienne ${ }^{167}$. Les sépultures mises au jour à Noisy-le-Grand ne semblent pas antérieures au $\mathrm{VI}^{\mathrm{e}}$ siècle et les stèles ne semblent présentes que dans celles datées entre le $\mathrm{VI}^{e}$ et le début du VIII ${ }^{e}$ siècle ${ }^{168}$. La période d'utilisation des stèles semble, comme dans la région voisine, se réduire pour l'essentiel aux seuls $\mathrm{VI}^{\mathrm{e}}$ et $\mathrm{VII}^{\mathrm{e}}$ siècles. L'examen comparatif des stèles de Gaillon avec la classification chronologique des stèles picardes permettrait de les dater de la période 500-650 $0^{169}$. L'usage disparaît totalement, dans le Vexin comme en Picardie, au milieu du VII ${ }^{\mathrm{e}}$ siècle ${ }^{170}$.

\section{PRODUCTIONS, USAGES SYMBO- LIQUES ET REMPLOIS OPPORTUNISTES}

Alors que l'épitaphe demeure, en Île-de-France, un phénomène essentiellement urbain ou périurbain lié aux élites lettrées, la stèle anépigraphe est largement diffusée dans les campagnes. L'utilisation de mêmes modules au sein des mêmes nécro-

158. BARAT 2007, p. 88.

159. Une plaque-boucle triangulaire de type monobloc et une seconde à cinq bossettes et décor natté (types 161 et 174 de LEgOUX, PÉRIN, VALLET 2016) figurent parmi le mobilier attribué à cette découverte (NAUDET et al. 2001, p. 107 et documentation inédite conservée au service départemental d'archéologie du Val-de-Marne).

160. BARAT 2007, p. 185.

161. Ibid., p. 229-231.

162. Sirat, Vieillard-Troiekourouff, Chatel 1984, p. 16.

163. BARAT 2007, p. 338-339.

164. Sirat 1971, p. 132; Wabont 2006, p. 122.

165. RÉGNARD 2002, p. 57.

166. ID. 2001.

167. MAzEAu 2006.

168. Le Forestier 2015, p. 194.

169. RÉGnARd 2001, p. 199-202; Flèche Mourgues 1992 et 1998.

170. Flèche Mourgues 1992, p. 51 et tableau IV; Flèche Mourgues 1998, p. 113-114; RÉGNARD 2002, p. 59-60. poles permet d'estimer que les stèles pouvaient ponctuellement et localement être réalisées en série, probablement par un même artisan. L'homogénéité des stèles de Santeuil à croix fleuronnées semble bien indiquer une pratique locale et probablement le recours à des personnes spécialisées, des ateliers locaux qui, à défaut d'une production de série comme pour les sarcophages, pouvaient être régulièrement amenés à réaliser ces monuments funéraires. Le statut de ces individus est difficile à établir mais, s'ils n'étaient pas liés à la mise en carrière des sites antiques, il est possible qu'ils étaient partie prenante dans la réalisation ou l'importation des sarcophages. Faute d'analyses pétrographiques, il est impossible d'établir, comme en Picardie ${ }^{171}$, la carrière d'origine des monuments. L'exemplaire de Genainville démontre toutefois que les blocs n'étaient pas taillés près des lieux d'inhumations mais que cette activité pouvait se faire sur le site de production. La stèle achevée était ensuite acheminée vers la nécropole.

L'hypothèse d'une résurgence de pratiques païennes locales pour les stèles à cavité n'est guère recevable. Les cavités n'apparaissent que tardivement, vers la fin du vie siècle, sur des stèles de formes différentes, alors que les plus anciennes, mises au jour dans les mêmes nécropoles, sont datées de la fin du $\mathrm{V}^{\mathrm{e}}$ et du début du $\mathrm{vI}^{\mathrm{e}}$ siècle $^{172}$. Cette apparition tardive semble donc exclure une survivance païenne de rites locaux, d'autant que des exemples similaires ont été observés dans l'Oise ou dans l'Aisne. Un autre argument invalide l'hypothèse d'une explication strictement païenne, c'est la coexistence de cavités aux côtés de motifs géométriques à Maule ${ }^{173}$. Une utilisation volontaire de motifs à symbolique a priori chrétienne en parallèle aux cavités est avérée. La reproduction à l'identique des motifs ornant chaque face (croix grecque et cavité) des deux stèles de modules comparables découvertes à Santeuil ${ }^{174}$, exclut la réutilisation opportuniste d'un monument remployé. Il s'agit d'une composition intentionnelle, pensée dès l'origine.

Le chrisme, une croix monogrammatique, les lettres apocalyptiques ou la représentation de colombes, sont significatifs d'une volonté délibérée d'afficher une représentation chrétienne. La représentation du chrisme constantinien est cependant rare. Présent sur les trois épitaphes de Melanius, Barbara et Theudote (fig. 8, no 1, 2 et 3), le chrisme est absent des stèles anépigraphes répertoriées en Île-de-France, alors qu'il peut être présent sur certaines stèles des régions voisines. Comme on l'a vu, les motifs rayonnants représentés sur plusieurs stèles ne symbolisent sans doute pas le chrisme, bien qu'une intention des lapicides en ce sens ne puisse être totalement écartée.

La représentation du Christ à Ableiges, la croix latine monogrammatique à Fresnes ou les lettres apocalyptiques utilisées sur un fragment de stèle à Guiry-en-Vexin sont rares. Les colombes ne sont parfaitement identifiables que sur l'épitaphe de Barbara et potentiellement sur une stèle d'Ableiges ${ }^{175}$ (fig. $6 n^{\circ} 3$ ). Les personnages sont rares. Ils ont pu être interprétés comme des orants sur deux stèles à Banthelu ${ }^{176}$ et à

171. Flèche Mourgues 1995, p. 141-142.

172. ID. 1998, p. 114.

173. Sirat, Vieillard-Troïekouroff, Chatel 1984, nº 147.

174. Couverture des sépultures no 106 et 112-113 (MAZEAU 2006).

175. Sirat 1966, pl. II, fig. 32.

176. Id. 1970, p. 98 ; Sirat, Vieillard-TroÏekouroff, Chatel 1984, nº 50. 
Maule ${ }^{177}$. La présence d'une représentation de poisson, associée à la mention pax sur une stèle isolée à Septeuil ${ }^{178}$ semble bien manifester la symbolique du christ, mais sa découverte hors contexte funéraire ne permet pas de déterminer l'origine de cette représentation.

La variété apparente des croix (grecque, latine, fleuronnée, processionnelle, pattée) ne masque pas la sobriété des représentations. La symbolique chrétienne des pseudo-croix de Malte réalisées au compas sur une dizaine de stèles peut être contestée. Seuls décors représentés sur ces stèles, ces croix ne sont pourtant jamais associées, comme sur les sarcophages de plâtre, à d'autres représentations géométriques. Les représentations sur ces stèles apparaissent comme des manifestations qui se distinguent des stèles non décorées et pourraient correspondre à une évolution vers des décors de motifs géométriques. L'importance de ces derniers pourrait-elle correspondre à une évolution chronologique, depuis des motifs à symbolique chrétienne jusqu'à des représentations plus géométriques? L'exactitude de la représentation ne semble plus toujours l'objectif privilégié. La symbolique chrétienne de la stèle d'Épône, par exemple, devient ainsi contestable. Ici, le souci d'esthétique semble avoir été davantage recherché, le motif ornant quasiment l'intégralité de l'espace. Comme pour les sarcophages de plâtre, les décors de rosaces ne font guère référence à une symbolique chrétienne, qui était assez clairement affichée sur les épitaphes ou les stèles plus anciennes, au $\mathrm{V}^{\mathrm{e}}$ siècle et probablement encore au début du VII ${ }^{\mathrm{e}}$ siècle.

Plusieurs stèles ont pu être remployées en couverture de sépultures. Ce remploi avait-il toujours une dimension symbolique pour les personnes assistant aux funérailles ou pour les défunts? Le remploi de stèles, face gravée de motifs chrétiens tournée vers le défunt, a pu être observé en couverture des sépultures no 78, 80 et 82 de Gaillon-sur-Montcient mais également sur les sarcophages de plâtre des tombes no 102 et 103 de Fresnes (fig. 12). Les stèles ont aussi été découvertes avec la face gravée tournée vers l'extérieur. C'est le cas des sépultures $\mathrm{n}^{\circ} 112$ et 113 de Santeuil (fig. 13) et de la sépulture $\mathrm{n}^{\circ} 79$ de Gaillon-sur-Montcient (fig. 14). Ailleurs, la stèle pouvait être incomplète ou installée, avec la face décorée d'une croix pattée processionnelle (fig. 6, n 6 ) tournée vers l'intérieur, comme support de la couverture de la sépulture no 81 de Gaillon-surMontcient ${ }^{179}$, mais la stèle (et donc la croix) était positionnée à l'envers. Ici, l'intérêt du remploi était d'obtenir une surface horizontale suffisamment régulière pour assurer le support de la couverture et non de se soucier du sens de la représentation sur cette stèle dont la partie supérieure, plutôt triangulaire, ne pouvait convenir à cet usage. D'autres, présentant des décors chrétiens, ont simplement été mises au jour dans les comblements. Trois exemplaires ont ainsi été découverts rejetés dans le remblai de la sépulture no 178 de Gaillon-sur-Montcient. Des stèles non décorées étaient pareillement utilisées dans le coffrage en couverture des inhumations, comme la sépulture no 95 de Gaillon. Quelques sépultures de Noisy-le Grand remployaient plusieurs stèles sans décors en couverture (fig. 15). Il semble

177. Sirat, Vieillard-Troïekouroff, Chatel 1984, no 133.

178. BARAT et al. 2007, p. 335.Ce type de représentation pourrait aussi correspondre à une influence germanique (GAUTHIER 1975, nº 135, p. 353).

179. RÉgnard 2001, p. 99-100, pl. 62; RÉGnARD 2002, p. 44; RÉGNARD, LANGLOIS, VANPEENE 2002, p. 71.

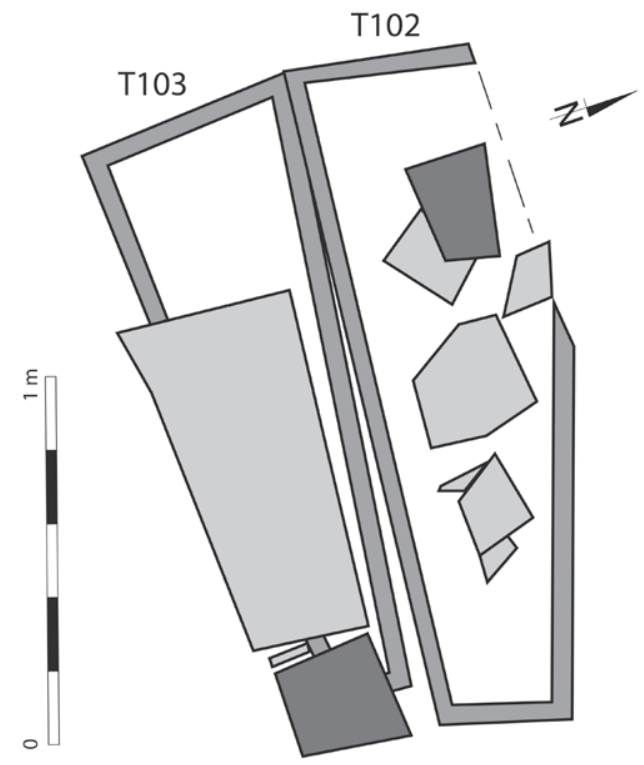

Fig. 12 Relevé des couvertures des sarcophages de plâtre des sépultures 102 et 103 de la nécropole de Fresnes (DAO d'après BOUCHER 1975, pl. 24 et 25).

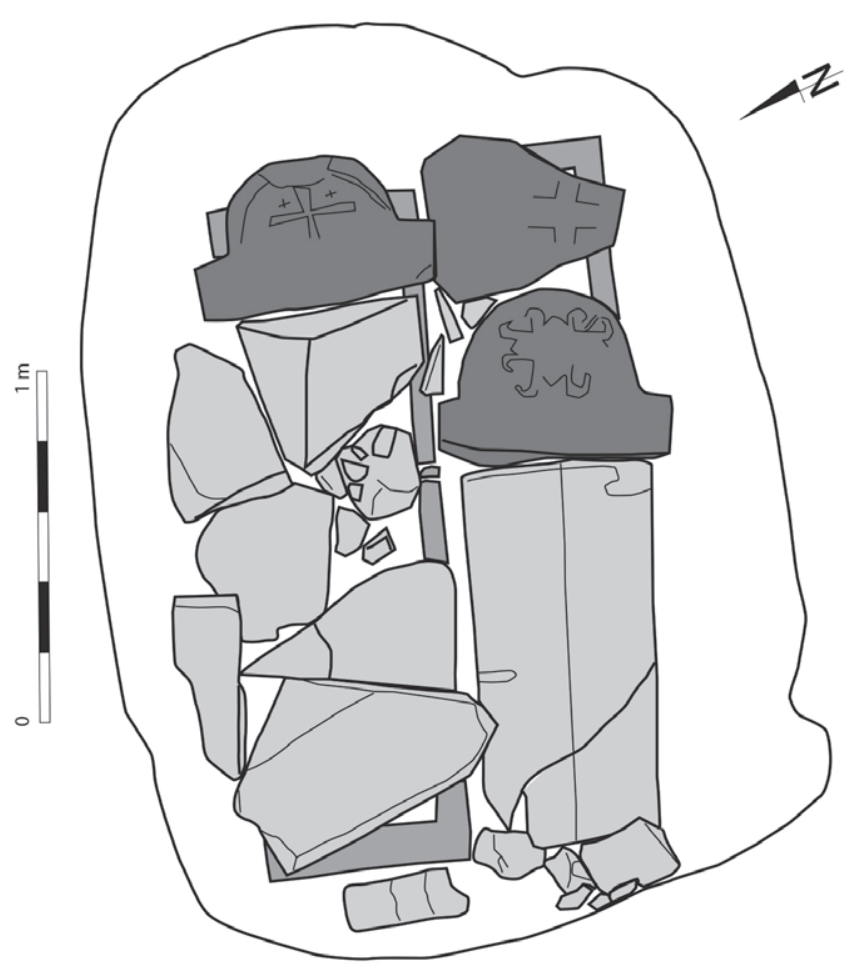

Fig. 13 Relevé des couvertures des sarcophages bi-partites des sépultures 112 et 113 de la nécropole de Santeuil. Les stèles remployées apparaissent en grisé foncé (DAO d'après MAZEAU 2006, p. 58, pl. 27).

donc que la disposition des décors des stèles remployées puisse être tout à fait fortuite et que l'objectif privilégié soit surtout l'opportunité du remploi de pierres plates facilement adaptables aux couvertures ou au coffrage des sépultures. Tout porte à penser que seul compte l'aspect pratique du monolithe et non la visibilité du signe, même si l'on ne peut totalement exclure que la charge symbolique ait pu jouer un rôle. 


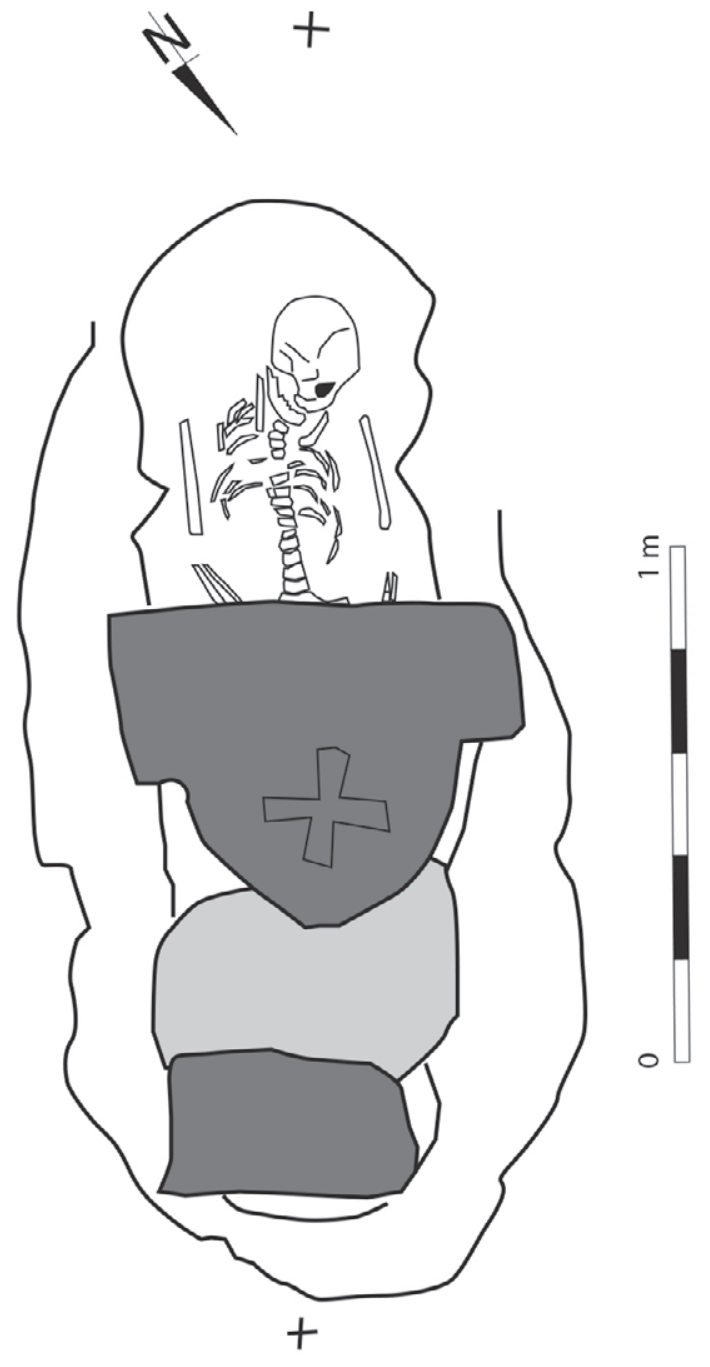

Fig 14 Relevé des couvertures de la sépulture 79 de Gaillon-surMontcient (DAO d'après RÉGNARD, LANGLOIS 1997, p. 97).

Peut-on déduire le statut social des inhumés ou des personnes ayant organisé les funérailles à partir de la présence de tels monuments en pierre? La question ne se pose pas pour une épitaphe, qui nécessite la maîtrise de l'écrit, très peu répandue à cette période en dehors des élites. Elle peut être davantage posée pour une simple stèle anépigraphe, décorée ou non. Le matériau utilisé, la taille et les décors sommaires ne semblent pas des obstacles financièrement insurmontables, mais l'existence d'ateliers de production comme d'un marché put, comme pour les sarcophages, déboucher sur des tarifs différenciés. La quasi-absence de découvertes de stèles en position primaire ne permet pas de comparer celles-ci au mobilier accompagnant le défunt pour qui le monument fut préparé. Il faut cependant

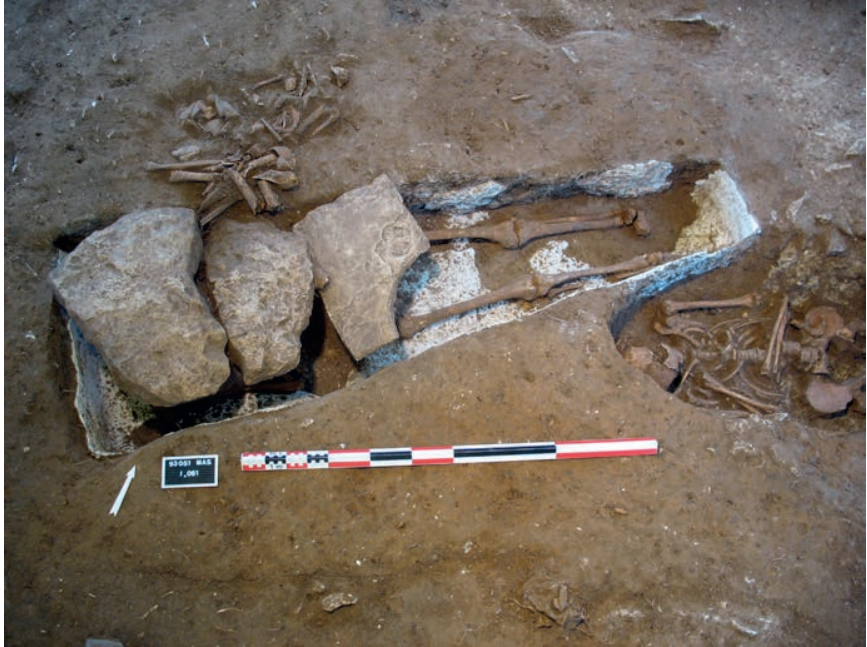

Fig. 15 Sépulture en coffrage de plâtre $(I, 061)$ recouverte de stèles remployées découvertes à Noisy-le-Grand sur le site des Mastraits (cl. C. Le Forestier, Inrap).

noter le faible nombre de découvertes dans les cimetières mérovingiens, qui pourrait être mis en regard du mobilier métallique mis au jour dans les mêmes nécropoles. Si l'on peut déplorer une part non négligeable de destructions de stèles, celles-ci peuvent être comparées aux pillages du mobilier dans les tombes. À titre d'exemple, la nécropole récemment fouillée des Mastraits à Noisy-le-Grand a livré, pour 219 inhumations mérovingiennes, 6 fibules, 17 plaque-boucles (dont deux hors contexte) et 2 scramasaxes. Ce faible taux de mobilier métallique peut être comparé aux 23 stèles découvertes en remplois ou aux 131 sarcophages moulés ${ }^{180}$, dont l'usage était peut-être plus répandu. Sans relations restituables entre le mobilier funéraire et les stèles, il est toutefois difficile de conclure sur cet aspect. L'une des deux stèles mises au jour à L'Haÿ-lesRoses (fig. 7) aurait été mise au jour à la tête d'une inhumation associée à une plaque-boucle. Seules deux plaque-boucles et deux stèles auraient été découvertes dans cette petite nécropole d'une soixantaine de sépultures.

Comment interpréter l'arrêt de l'utilisation de stèles anépigraphes, vraisemblablement dans le courant du vire siècle? Il est peu probable qu'il s'agisse de la disparition d'un savoir-faire. C'est également à cette période que l'usage des sarcophages de pierre ou de plâtre décorés se termine, que l'inhumation habillée n'est plus pratiquée et que les dépôts funéraires s'interrompent. La fin de l'utilisation des stèles monolithiques accompagne donc naturellement les changements qui affectent les pratiques funéraires dans le courant du viI ${ }^{e}$ siècle.

180. Le Forestier 2015 


\section{BIBLIOGRAPHIE}

ArdouIn S.

2001, «Une inscription funéraire chrétienne provenant de la chapelle Saint-Frambour à Ivry-sur-Seine (Val-de-Marne)", Archéologie Médiévale, 2000-2001, t. 30-31, p. 39-65.

2006, «Quelques témoignages archéologiques des usages funéraires chrétiens mérovingiens dans le sud-est parisien", CLIO 94, Bulletin du comité de liaison des sociétés d'histoire et d'archéologie du Val-de-Marne, $\mathrm{n}^{\circ}$ 24, Actes du colloque «La vie religieuse dans le sud-est parisien des origines à nos jours », p. 52-68.

2008, L'apport des données archéologiques pour la connaissance de la situation religieuse de l'Ile-de-France (IV -VIII $^{e}$ siècle), Mémoire de Master Recherche (sous la dir. de J. Burnouf et P. Perin), Université de Paris 1 Panthéon-Sorbonne, 209 p.

À paraître, «Les épitaphes et stèles funéraires d'île-de-France (IV ${ }^{e}-V_{\text {VIII }}{ }^{e}$ siècle)", dans Dierkens A., Pettiau H. (dir.), Épigraphie, archéologie et histoire de l'Antiquité tardive et du haut Moyen Âge (IV ${ }^{e}-I X^{e}$ siècle), Actes des XVI Journées Lotharingiennes - XXXI Journées internationales d'archéologie mérovingienne de l'Afam, Luxembourg/Arlon (14-17 octobre 2010), Mémoires de l'Afam/Cludem, parution prévue en 2018.

Augereau A.

1995, «Saint-Sauveur-lès-Bray. Le Port aux Pierres", dans Blanchet J.-C. (dir.), Bilan scientifique 1995 Île-de-France, Paris, Service régional de l'archéologie d'Île-de-France, p. 78-79.

BARAT Y.

2007, Carte archéologique de la Gaule, Yvelines (78), Paris, Académie des Inscriptions et Belles-Lettres.

Barat Y. en coll. avec LANGlois M., BRICON D.

2001, «Habitats et nécropoles du haut Moyen Âge en vallée de Vaucouleurs (sites de Septeuil et Villette, Yvelines)", Revue archéologique du Centre de la France, t. 40, p. 133-165.

BenHaddou S. (dir.)

1990, Melun au temps de Rome, Catalogue d'exposition, Melun, Ville de Melun.
Boucher M.

1975, Rapport des fouilles de sauvetage effectuées en 1975 sur le site du chemin de la Tour aux Chartiers (commune de Fresnes), Fresnes, Société archéologique de Fresnes.

Busson D.

1998, Carte archéologique de la Gaule, Paris (75), Paris, Académie des Inscriptions et Belles-Lettres.

Delattre V., Magnan D.

1993, «Meaux», dans DepraetèreDargery M., Petit M. (dir.), L'îlede-France de Clovis à Hugues Capet du $V^{e}$ au $X^{e}$ siècle, Catalogue d'exposition, Musée archéologique départemental du Val-d'Oise, Service régional de l'archéologie d'île-de-France, Cergy-Pontoise, Éditions du Valhermeil, p. 148-150.

1998, «De la limite sacrée à l'enceinte du Bas-Empire à Meaux", dans Delattre V., Magnan D. (dir.), Profane et sacré en pays meldois. Protohistoire Gallo-romain, Meaux, AMA-Musée Bossuet, p. 127-135.

Descombes F.

1985, Recueil des inscriptions chrétiennes de la Gaule antérieures à la Renaissance carolingienne, t. XV : Viennoise du Nord, Paris, Éditions du CNRS.

Dierkens A., Périn P.

2011, "Croyances de la Gaule mérovingienne. Du paganisme au christianisme en Gaule : les leçons de l'histoire et de l'archéologie funéraire", dans Dierkens A., Périn P. (coord.), Le baptême de Clovis, Religions et histoire, $n^{\circ} 41$, p. 42-51

Duval P.-M.

1960, Les Inscriptions antiques de Paris, 2 t., Paris, Imprimerie nationale.

Flèche Mourgues M.-P.

1992, "Caractéristiques des monuments sculptés du haut Moyen Âge dans le nord de la Gaule», Revue du Nord, t. LXXIV, $n^{\circ} 296$, p. 29-67.

1995, «La question des ateliers de sculpteurs du haut Moyen Âge en Picardie», Revue archéologique de Picardie, 3/4, p. 141-156.

1998, «La classification typo-chronologique des stèles funéraires en Picardie», dans Delestre X., Périn P. (dir.), La datation des structures et des objets du haut Moyen Âge: méthodes et résultats, Actes des XVe journées d'archéologie mérovingienne de Rouen, 4-6 février 1994, Saint-Germain-en-Laye, Mémoires de l'Association française d'archéologie mérovingienne, p. 105-115.

Frère S., Héron C.

1998, Tremblay-en-France, Château Bleu,

Document final de synthèse de sauvetage urgent, Bobigny, Département de la Seine-Saint-Denis.

Fossard D., Vieillard-Troïekouroff M., Chatel É.

1978, Recueil général des monuments sculptés en France pendant le haut Moyen Âge (IV $V^{e} X^{e}$ siècles), t. I : Paris et son département. Paris, Bibliothèque nationale.

Gauthier N.

1975, Recueil des inscriptions chrétiennes de la Gaule antérieures à la Renaissance carolingienne, t. 1 : Première Belgique, Paris, Éditions du CNRS.

Giraud E.

1929, «Un cimetière mérovingien sur les hauteurs d'Arcueil (Seine)", Revue anthropologique, no 39, p. 305-306.

Griffisch J.-N., Magnan D., Mordant D. 2008, Carte Archéologique de la Gaule, Seine-et-Marne (77), Paris, Académie des Inscriptions et Belles-Lettres, 2 vol.

Guadagnin R.

1988, «Le cimetière de la villa de Villiers-le-Sec», dans Cuisenier J., GUADAGNIN R. (dir.), Un village au temps de Charlemagne, moines et paysans de l'abbaye de Saint-Denis du vir siècle à l'an $\mathrm{Mil}$, Catalogue de l'exposition au Musée national des arts et traditions populaires (29 novembre 1988 - 30 avril 1989), Paris, Réunion des musées nationaux, p. 166-176.

Hirschfeld O., Zangemeister C. (éd.) 1899-1916, Corpus Inscriptionum Latinarum, XIII, Inscriptiones trium Galliarum et Germaniarum Latinae: Inscriptiones Aquitaniae et Lugudunensis, Berolini, Apud G. Reimerum.

Le Blant E. (éd.)

1856, Les inscriptions chrétiennes de la Gaule antérieures au VIII eiècle, $t$. 1 : Provinces gallicanes, Paris, Imprimerie impériale. 
1865, Les inscriptions chrétiennes de la Gaule antérieures au viIr siècle, t. 2 : Les sept provinces, Paris, Imprimerie impériale.

Le Forestier C.

2015, «La nécropole du haut Moyen Âge des Mastraits à Noisy-le-Grand (Seine-SaintDenis)», dans Hadjouis D., Ardouin S. (dir.), Dynamique des peuplements, modes d'habitat et influences culturelles dans le Sud-Est de Paris du Néolithique ancien à la période Moderne, Actes du colloque de Créteil, 3 et 4 juin 2010, Paris (Supplément à la Revue archéologique d'île-de-France, 3), p. 191-221.

Legoux R., Périn P., VAllet F.

2016, Chronologie normalisée du mobilier funéraire mérovingien entre Manche et Lorraine, Bulletin de liaison de l'Association française d'archéologie mérovingienne, hors série, $4^{\mathrm{e}}$ éd. revue et corrigée.

MacMullen R.

1998, Christianisme et paganisme du IV ${ }^{e}$ au VIII siècle, Paris, Les Belles Lettres (trad. de Christianity and Paganism in the Fourth to Eighth Centuries, Londres, 1996).

Mazeau A.

2006, «La nécropole mérovingienne de Santeuil (Val-d'Oise)», Bulletin archéologique du Vexin français, no 38, p. 23-99.

Mitard P.-H.

1973, «Le remploi de blocs antiques pour la confection de sarcophages et de stèles à l'époque mérovingienne, l'exemple de Genainville», Bulletin archéologique du Vexin français, no 9, p. 110-114.

Mousset F.

1999, Ivry-sur-Seine, Place Parmentier-îlot 2, Rapport de fouille archéologique, Villejuif, Laboratoire départemental d'archéologie du Val-de-Marne, déposé à la Drac Île-de-France, 3 vol.

NAudet F.

2001, Carte archéologique de la Gaule, Val-de-Marne (94), Paris, Académie des Inscriptions et Belles-Lettres.

2004, Carte archéologique de la Gaule, Essonne (91), Paris, Académie des Inscriptions et Belles-Lettres.

Pachtere F.-G. de

1912, Paris à l'époque gallo-romaine. Étude faite à l'aide des papiers et des plans de Th. Vacquer, Paris, Imprimerie nationale.
PÉRIN P.

1987, «Des nécropoles romaines tardives aux nécropoles du haut Moyen Âge», Cahiers archéologiques, $\mathrm{n}^{\circ} 35$, p. 9-30.

PÉRIN P. (dir.)

1985, Collections mérovingiennes (Catalogues d'art et d'histoire du musée Carnavalet), Paris, Musée Carnavalet.

Piétri L.

1985, «Les inscriptions chrétiennes durant le haut Moyen Âge» dans PÉRIN P. (dir.), Collections mérovingiennes (Catalogues d'art et d'histoire du musée Carnavalet), Paris, Musée Carnavalet, p. 554-562.

Poulain C.

1984, «Les stèles mérovingiennes en Champagne-Ardenne et dans le département de l'Aisne», Bulletin de la Société archéologique Champenoise, 1984-4, p. 15-26.

Prévot F.

1997, Recueil des inscriptions chrétiennes de la Gaule antérieures à la Renaissance carolingienne, Aquitaine Première, Paris, Éditions du CNRS.

Rebillard E.

2003, Religion et sépulture. L'Église, les vivants et les morts dans l'Antiquité tardive, Paris, Éditions de l'EHESS.

RÉGNARD S.

1996, «Gaillon-sur-Montcient (nécropole de La Garenne)", Bulletin archéologique du Vexin français, n² 29, p. 101-104.

2001, Gaillon-sur-Montcient (Yvelines). Lieu-dit la Garenne. Nécropole mérovingienne. Campagne archéologique 1996. Fouilles du secteur 3, Document Final de Synthèse, Cravo, déposé à le Drac Île-deFrance.

2002, "La nécropole mérovingienne de La Garenne à Gaillon-sur-Montcient (Yvelines)", dans Trombetta P.-J., Régnard S. dir., Mérovingiens et Carolingiens en Île-de-France. Découvertes archéologiques. Catalogue de l'exposition de Mantes-la-Jolie du 6 octobre 2002 au 31 mars 2003, Paris, Somogy, p. 41-63.

RÉGNARD S., LANGLOIS M.,

1997, Gaillon-sur-Montcient (Yvelines). Nécropole mérovingienne de La Garenne, Rapport de fouille. Versailles, Cravo/ Sady, 2 vol., déposé à le Drac Île-deFrance.

RÉGnard S., LANGlois M., VANPEene N. 2002, «Objets de Gaillon-sur-Montcient (nécropole de La Garenne)» dans
Trombetta P.-J., Régnard S. (dir.), Mérovingiens et Carolingiens en Île-deFrance. Découvertes archéologiques, Catalogue de l'exposition de Mantes-laJolie du 6 octobre 2002 au 31 mars 2003, Paris, Somogy, p. 64-71.

SALIN E.

1952, La civilisation mérovingienne d'après les sépultures, les textes et le laboratoire. Deuxième partie: les sépultures, Paris, Picard.

Séguier J.-M., Auxiette G., Bertin P., Pilon F.

2009, «La ferme et les stèles gallo-romaines $\mathrm{du}$ "Port aux Pierres" à Saint-Sauveurlès-Bray (Seine-et-Marne)», Revue archéologique d'Île-de-France, n², p. 147-170.

Sirat J.

1966, «Les stèles mérovingiennes du Vexin français», Bulletin archéologique du Vexin français, t. 2, 1966, p. 73-83.

1970, «Les stèles mérovingiennes du Vexin français. Inventaire complémentaire", Bulletin archéologique du Vexin français, $\mathrm{n}^{\circ} 6, \mathrm{p} .95-103$.

1973, «Les stèles mérovingiennes de la région parisienne», Bulletin archéologique du Vexin français, ${ }^{\circ}$ 9, p. 115-117.

1980, «Le cimetière mérovingien de Stors, Commune de Mériel (Val-d'Oise)", Bulletin archéologique du Vexin français, $\mathrm{n}^{\circ} 16$, p. 111-117.

1981, «Les cimetières mérovingiens de Genainville (Val-d'Oise)», Bulletin archéologique $d u$ Vexin français, $\mathrm{n}^{\circ} 17$, p. 97-108.

1993, «La nécropole de Santeuil (Val-d'Oise)», dans DepraetereDargery M., Petit M. (dir.), L'îlede-France de Clovis à Hugues Capet du $V^{e}$ au $X^{e}$ siècle, Catalogue d'exposition, Musée archéologique départemental du Val-d'Oise, Service régional de l'archéologie d'Île-de-France, Cergy-Pontoise, Éditions du Valhermeil, p. 220-221.

Sirat J., Vieillard-Troïekouroff M., Chatel E.

1984, Recueil des monuments sculptés en France pendant le haut Moyen Âge (IV ${ }^{e-}$ $\mathrm{X}^{e}$ siècles), t. III : Le Val-d'Oise et les Yvelines, Paris, CTHS.

Treffort C.

1996, L'église carolingienne et la mort. Christianisme, rites funéraires et pratique, Lyon, Centre interuniversitaire d'histoire et d'archéologie médiévales / Presses universitaires de Lyon (Collection 
d'histoire et d'archéologie médiévales, no 3).

Vieillard-Troïekouroff M., Frossard D., Chatel E., Lamy-Lassalle C.

1960, «Les anciennes églises suburbaines de Paris ( $\mathrm{IV}^{\mathrm{e}}-\mathrm{X}^{\mathrm{e}}$ siècle)», Paris et Île-deFrance, Mémoires de la Fédération des Sociétés historiques et archéologiques de Paris et de l'̂̂le-de-France, no 11, p. 17-282.
WABONT M.

2006, «L'époque mérovingienne», dans Wabont M., Abert F., Vermeersch D., Carte archéologique de la Gaule, Val d'Oise (95), Paris, Académie des Inscriptions et Belles-Lettres, p. 117-126.

Wabont M., Abert F., Vermeersch D.

2006, Carte archéologique de la Gaule, Val d'Oise (95), Paris, Académie des Inscriptions et Belles-Lettres.
Young B. en coll. avec PÉrin P.

1991, "Les nécropoles (III ${ }^{\mathrm{e}}$-VIII ${ }^{\mathrm{e}}$ siècle)", dans Duval N., Naissance des Arts chrétiens. Atlas des monuments paléochrétiens de la France, Paris, Imprimerie nationale, p. 94-121. 